\title{
Quantization of massive Dirac billiards and unification of nonrelativistic and relativistic chiral quantum scars
}

\author{
Min-Yue Song, ${ }^{1} \mathrm{Zi}$-Yuan $\mathrm{Li},{ }^{1}$ Hong-Ya Xu, ${ }^{2}$ Liang Huang, ${ }^{1,{ }^{*}}$ and Ying-Cheng Lai ${ }^{2,3}$ \\ ${ }^{1}$ School of Physical Science and Technology, and Key Laboratory for Magnetism and Magnetic Materials of MOE, \\ Lanzhou University, Lanzhou, Gansu 730000, China \\ ${ }^{2}$ School of Electrical, Computer, and Energy Engineering, Arizona State University, Tempe, Arizona 85287, USA \\ ${ }^{3}$ Department of Physics, Arizona State University, Tempe, Arizona 85287, USA
}

(Received 1 July 2019; published 3 October 2019)

\begin{abstract}
Quantum scars constitute one of the fundamental pillars in the traditional field of nonrelativistic quantum chaos. In relativistic quantum systems, chiral scars have been discovered recently whose wave functions concentrate on odd periodic orbits and break the time-reversal symmetry. We develop a theoretical framework to unify the scarring phenomena in nonrelativistic and relativistic quantum systems, which were previously thought to be distinct. In particular, we exploit massive Dirac billiard systems and derive semiclassical quantization rules to bridge the two opposite limits: the massless Dirac case and the large mass regime where the system effectively degenerates into one governed by the Schrödinger equation. A nontrivial phase is uncovered, which depends on the mass, the wave number, and the angle of reflection, and we demonstrate that this phase plays a key role in transforming the chiral scars and in bridging the relativistic and nonrelativistic quantum scars. In the large-mass limit, time-reversal symmetry is restored, as evidenced by a spectral analysis.
\end{abstract}

DOI: 10.1103/PhysRevResearch.1.033008

\section{INTRODUCTION}

In quantum chaos, a branch of physics that studies the quantum manifestations of classical chaos [1,2], a fundamental topic is scarring. Quantum scars are referred to as the relatively high concentrations of the wave function about certain unstable periodic orbits of the system dynamics in the classical limit. In such a system, e.g., a two-dimensional (2D) stadium billiard, the classical phase space is ergodic and the density of the trajectories is uniform in the physical space. Intuition would suggest that the quantum wave-function distributions be uniform as well. It is against this intuition which makes any nonuniform wave-function distribution surprising. Historically, highly nonuniform concentrations of the quantum eigenwave functions about classical periodic orbits were first discovered by McDonald and Kaufman [3,4], which were later called "quantum scars" by Heller [5]. Since then, quantum scars have fascinated physicists [4,6-39].

Experimental separation of graphene about one and half decades ago [40-46] led to the tremendous development of condensed-matter systems whose quasiparticles obey the laws of relativistic quantum mechanics governed by the Dirac equation or its generalizations. This in turn has stimulated interests in the relativistic quantum manifestations of classical chaos (see Refs. [47,48] and references therein). A seminal

\footnotetext{
*huangl@1zu.edu.cn

Published by the American Physical Society under the terms of the Creative Commons Attribution 4.0 International license. Further distribution of this work must maintain attribution to the author(s) and the published article's title, journal citation, and DOI.
}

contribution was made by Sir Michael Berry and his collaborator, who studied the spectral properties of the eigenenergies of the massless Dirac equation for the chaotic neutrino billiard [49]. However, efforts in relativistic quantum chaos were largely sporadic before the development of two-dimensional Dirac materials. On the subject of scarring, relativistic quantum scars were uncovered in graphene systems [50] and observed experimentally [51]. A peculiar class of relativistic quantum scars in chaotic massless Dirac billiard systems was discovered in which the difference between the accumulated phase along a counterclockwise orbit in a complete cycle and that along the clockwise cycle is $\pi$ modulo $2 \pi$. These are chiral scars [52] that break the time-reversal symmetry $[49,53,54]$ and have no counterpart in nonrelativistic quantum systems.

In this paper, we develop a theory to unify scarring in nonrelativistic and relativistic quantum systems through analyzing the phase behaviors of the eigenstates of massive Dirac billiard systems. In all existing studies of relativistic quantum scars [50-53,55], the quasiparticles have zero mass so the energy-momentum dispersion relation is linear. This picture describes well the physics of quasiparticles in a variety of two-dimensional (2D) Dirac material systems such as an ideal graphene sheet in the low-energy regime or the surface electrons of a topological insulator $[56,57]$. In physical reality, because of the necessity of opening band gaps for device applications and the realization of experimental schemes for a gate-controlled, continuously tunable band gap [58], the concept of massless quasiparticles is no longer sufficient, rendering necessary a description based on massive Dirac particles. The physics of such quasiparticles remains relativistic quantum with the underlying equation being the massive Dirac 
equation. Scarring in relativistic quantum systems described by the Dirac equation with a finite mass is by itself an open problem. From the fundamental standpoint of unification, the massive Dirac equation has two limiting regimes: (a) zero mass and (b) a large finite mass, where the former describes the behavior of massless Dirac fermions and, for the latter, if the mass is sufficiently large, the first component of the spinor effectively describes the corresponding Schrödinger system. Studying quantum scarring of massive Dirac particles can thus unify the conventional, nonrelativistic quantum scars and chiral scars in massless Dirac fermion systems.

Our key finding in developing a scar-unifying theory is that the mass term in the Dirac equation leads to a dynamical phase that depends not only on the angles but also on the mass and wave number. This enables us to derive a semiclassical formula for the phase accumulation and, consequently, to fully predict the emergence and properties of scars. Another result is that the dynamical phase and the semiclassical formula can be used to understand the spectral properties of massive Dirac fermions. The significance of our findings is as follows. Nonrelativistic quantum scars have become a quite well-studied topic in quantum chaos while the discovery of relativistic quantum scars in massless Dirac systems was relatively recent. Our work reveals that these two types of scarring phenomena, characteristically distinct properties notwithstanding, are different aspects of the same entity: scars in massive Dirac systems with the dynamical phase as the unifying thread.

\section{MASSIVE DIRAC BILLIARD}

Consider a $2 \mathrm{D}$ spin- $1 / 2$ particle of mass $m$ in a billiard region $\mathcal{D}$, where the particle moves freely inside the billiard and reflects at the boundary. The massive Dirac equation is

$$
\left[-i \hbar c(\hat{\boldsymbol{\sigma}} \cdot \nabla)+m c^{2} \hat{\sigma}_{z}+V(\mathbf{r}) \hat{\sigma}_{z}\right] \psi(\mathbf{r})=E \psi(\mathbf{r}),
$$

where $\psi(\mathbf{r})=\left[\psi_{1}(\mathbf{r}), \psi_{2}(\mathbf{r})\right]^{\mathrm{T}}$ is the two-component spinor wave function, $\hbar$ is the reduced Planck constant, $c$ is the speed of light (or the Fermi velocity), $\hat{\boldsymbol{\sigma}}=\left(\sigma_{x}, \sigma_{y}\right)$ and $\hat{\sigma}_{z}=$ $\sigma_{z}$ are the Pauli matrices, and $E=\sqrt{(\hbar c k)^{2}+\left(m c^{2}\right)^{2}}$ is the energy. The confinement potential $V(\mathbf{r})$ is zero inside $\mathcal{D}$, and infinite outside $\mathcal{D}$. For convenience, we use the unit convention $\hbar=c=1$. Hermiticity of the Hamiltonian requires that the outward current vanish, which leads to the boundary condition $\psi_{2} /\left.\psi_{1}\right|_{\partial D}=i \exp \{i \alpha\}$, where $\alpha$ is the angle of the normal vector of the boundary measured from the positive $x$ direction, as shown in Fig. 1(a). For $m \rightarrow 0$, the massive Dirac billiard degenerates to one described by the massless Dirac equation. For $m \rightarrow \infty$, we have $\psi_{2} \rightarrow 0$ homogeneously inside the billiard $\mathcal{D}$ and the first component $\psi_{1}(\mathbf{r}, t)$ satisfies the Schrödinger equation with the Dirichlet boundary condition $\left.\psi_{1}\right|_{\partial D}=0-$ a nonrelativistic quantum billiard (see Appendix A for more details).

To calculate a large number of states for chaotic massive Dirac billiard, we generalize the conformal mapping method previously developed for the massless Dirac equation [52]. The starting point is the eigenfunctions $\psi_{l m}$ and the corresponding eigenwave number $\mu_{l m}$ of a circular massive Dirac billiard (see Appendix B), where a key parameter is

$$
\kappa \equiv \sqrt{\frac{1-m c^{2} / E}{1+m c^{2} / E}}=\sqrt{\frac{\sqrt{1+\hbar^{2} k^{2} / m^{2} c^{2}}-1}{\sqrt{1+\hbar^{2} k^{2} / m^{2} c^{2}}+1}},
$$

which plays a determining role in the dynamical phase. For a chaotic billiard with an analytic boundary that can be transformed from a circle, the massive Dirac equation can be solved in the circular domain in a modified form. By expanding the wave function in the base of $\left\{\psi_{l m}\right\}$, the problem can be converted to a standard matrix eigenequation, where the eigenvalues and eigenvectors can be used to yield the eigenwave number $k_{n}$ and eigenwave function $\Psi_{n}(\mathbf{r})$ of the original chaotic massive Dirac billiard system (see Appendix $\mathrm{C}$ for details). To be concrete, we consider a billiard with the boundary

$$
w(z)=\left(z+c_{1} z^{2}+c_{2} e^{i d} z^{3}\right) / \sqrt{1+2 c_{1}^{2}+3 c_{2}^{2}},
$$

where $|z|=1$ and the parameter values are $\left(c_{1}=0.49, c_{2}=\right.$ $d=0)$ and $\left(c_{1}=c_{2}=0.2, d=\pi / 3\right)$ for the heart-shaped and Africa billiards, respectively [59]. We calculate 40000 base eigenstates and use the first one third in our analysis to guarantee accuracy.
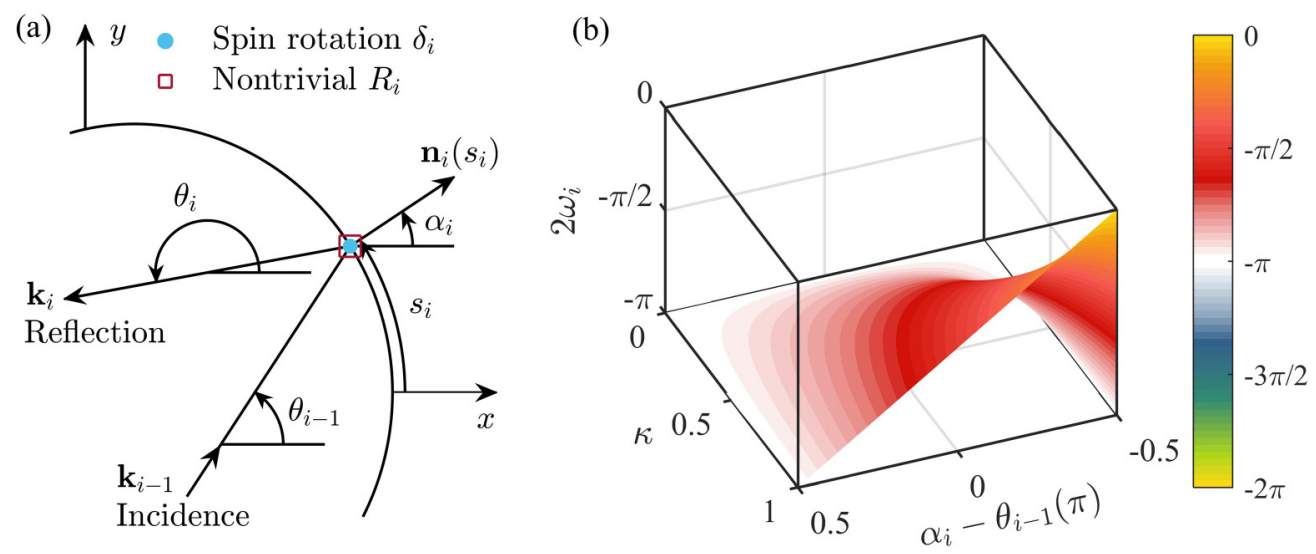

FIG. 1. Critical phases. (a) Incident and reflected local plane waves for the massive Dirac billiard. At the reflection point, there is a phase $\delta_{i}=\left(\theta_{i}-\theta_{i-1}\right) / 2=\alpha_{i}-\theta_{i-1}+\pi / 2$ due to spin rotation for both massless or massive Dirac fermions, and another phase in the nontrivial reflection coefficient $R_{i}: \arg \left(R_{i}\right)=2 \omega_{i}+\delta_{i}$ (see text). (b) Dependence of the dynamical phase $\left(2 \omega_{i}\right)$ on $\kappa$ and the angle $\left(\alpha_{i}-\theta_{i-1}\right)$. 


\section{QUANTIZATION FORMULA FOR SCARRING STATES IN MASSIVE DIRAC BILLIARD SYSTEMS}

In general, the condition for a scarring state to emerge is that, starting from any point on a periodic orbit, after traversing the orbit a complete cycle, the total accumulated phase is an integer multiple of $2 \pi$. For the massive Dirac billiard, for each reflection on the boundary, there is a phase

$$
\delta_{i}=\left(\theta_{i}-\theta_{i-1}\right) / 2=\alpha_{i}-\theta_{i-1}+\pi / 2
$$

due to spin rotation $[49,55]$. Using the plane wave analysis $[49,60]$ as illustrated in Fig. 1(a), we can derive that the reflection coefficient $R_{i}$ is no longer one, but contributes a nontrivial phase (see Sec. III A below): $\arg \left(R_{i}\right)=2 \omega_{i}+\delta_{i}$, where

$$
\omega_{i}=\arg \left(\kappa e^{-i\left(\alpha_{i}-\theta_{i-1}\right)}-i\right) .
$$

The complex functional profile of $\left(2 \omega_{i}\right)$ is shown in Fig. 1(b). Note that $\left(2 \omega_{i}\right)$ is asymmetric for positive and negative values of $\left(\alpha_{i}-\theta_{i-1}\right)$, except for $\kappa=0$ (the Schrödinger limit), indicating T-symmetry breaking for the massive Dirac billiard in general.

\section{A. Plane-wave analysis}

To develop a comprehensive theory for scars in chaotic massive Dirac billiard systems, we resort to plane-wave analysis, which was previously used to yield a detailed understanding of quantum scarring in massless Dirac billiards with a clear physical picture [49]. In the plane-wave representation, the spinor wave function of a positive energy state with a wave vector $\mathbf{k}$ of absolute value $k$ and angle $\theta$ with respect to the positive $x$ axis is given by

$$
\psi=\sqrt{\frac{E+m c^{2}}{2 E}}\left(\begin{array}{l}
e^{-i \theta / 2} \\
\kappa e^{i \theta / 2}
\end{array}\right) e^{i \mathbf{k} \cdot \mathbf{r}},
$$

where $\kappa$ is given by Eq. (2). The wave inside the domain can be written as the superposition of an incident wave and a reflected wave:

$$
\Psi^{\mathrm{I}}=\sqrt{\frac{E+m c^{2}}{2 E}}\left[\left(\begin{array}{l}
e^{-i \theta_{i-1} / 2} \\
\kappa e^{i \theta_{i-1} / 2}
\end{array}\right) e^{i \mathbf{k}_{i-1} \cdot \mathbf{r}}+R_{i}\left(\begin{array}{l}
e^{-i \theta_{i} / 2} \\
\kappa e^{i \theta_{i} / 2}
\end{array}\right) e^{i \mathbf{k}_{i} \cdot \mathbf{r}}\right],
$$

where $R_{i}$ is the reflection coefficient for the $i$ th reflection, and the incident and reflected wave vectors are $\mathbf{k}_{i-1}=\left(k \cos \theta_{i-1}, k \sin \theta_{i-1}\right)$ and $\mathbf{k}_{i}=\left(k \cos \theta_{i}, k \sin \theta_{i}\right)$, respectively. In the general case where the domain is confined by a finite potential $V$, the transmitted wave outside the domain is

$$
\Psi^{\mathrm{II}}=\frac{T_{i}}{\sqrt{2(V q-E K)}}\left(\begin{array}{c}
-i \lambda_{i 1} e^{-i \alpha_{i} / 2} \\
\lambda_{i 2} e^{i \alpha_{i} / 2}
\end{array}\right) e^{-q_{i} n} e^{i K_{i} s},
$$

where $T_{i}$ is the transmission coefficient, $K_{i}=k \sin \theta_{i-1}$, the attenuation coefficient in the normal direction is

$$
q_{i}=\sqrt{\left(V^{2}-E^{2}\right) /(\hbar c)^{2}+K_{i}^{2}},
$$

$\lambda_{i 1}=\sqrt{(V+E)\left(q_{i}-K_{i}\right)}, \lambda_{i 2}=\sqrt{(V-E)\left(q_{i}+K_{i}\right)}$, and $n$ and $s$ are the normal and tangential coordinates. Continuity of

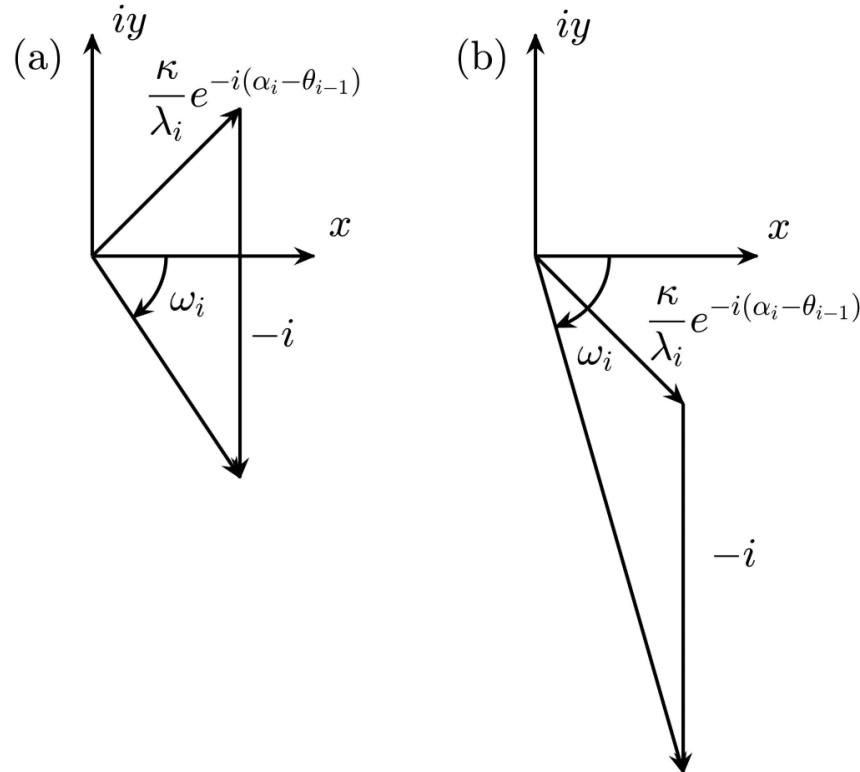

FIG. 2. Diagram for $\omega_{i}$ in the reflection coefficient. (a) For the case shown in Fig. 1. (b) For the reversed case.

the spinor wave function at the boundary requires

$$
\begin{aligned}
\sqrt{\frac{E+m c^{2}}{E}}\left(e^{-i \theta_{i-1} / 2}+R_{i} e^{-i \theta_{i} / 2}\right) & =-i \frac{\lambda_{i 1} T_{i} e^{-i \alpha_{i} / 2}}{\sqrt{V q-E K}}, \\
\sqrt{\frac{E+m c^{2}}{E}} \kappa \cdot\left(e^{i \theta_{i-1} / 2}+R_{i} e^{i \theta_{i} / 2}\right) & =\frac{\lambda_{i 2} T_{i} e^{i \alpha_{i} / 2}}{\sqrt{V q-E K}},
\end{aligned}
$$

which yields the reflection and transmission coefficients as

$$
\begin{aligned}
R_{i} & =\frac{\frac{\kappa}{\lambda_{i}} e^{i \theta_{i-1}}-i e^{i \alpha_{i}}}{e^{i \theta_{i-1}}-i \frac{\kappa}{\lambda_{i}} e^{i \alpha_{i}}}=i e^{i\left(\alpha_{i}-\theta_{i-1}\right)} \frac{\frac{\kappa}{\lambda_{i}} e^{-i\left(\alpha_{i}-\theta_{i-1}\right)}-i}{\frac{\kappa}{\lambda_{i}} e^{i\left(\alpha_{i}-\theta_{i-1}\right)}+i} \\
& =e^{i\left(2 \omega_{i}+\alpha_{i}-\theta_{i-1}+\pi / 2\right)}, \\
T_{i} & =2 C \sin \left(\omega_{i}+\alpha_{i}-\theta_{i-1}\right) e^{i\left(\omega_{i}+\left(\alpha_{i}-\theta_{i-1}\right) / 2-\pi / 2\right)} .
\end{aligned}
$$

As shown in Fig. 2, $\omega_{i}$ is given by

$$
\omega_{i}=\arg \left(\frac{\kappa}{\lambda_{i}} e^{-i\left(\alpha_{i}-\theta_{i-1}\right)}-i\right),
$$

or

$$
\tan \omega_{i}=-\frac{\frac{\kappa}{\lambda_{i}} \sin \left(\alpha_{i}-\theta_{i-1}\right)+1}{\frac{\kappa}{\lambda_{i}} \cos \left(\alpha_{i}-\theta_{i-1}\right)},
$$

and

$C=\sqrt{\frac{\left(E-m c^{2}\right)\left(V q_{i}-E K_{i}\right)}{E(V-E)\left(q_{i}+K_{i}\right)}}, \quad \lambda_{i}=\frac{\lambda_{i 2}}{\lambda_{i 1}}=\sqrt{\frac{(V-E)\left(q_{i}+K_{i}\right)}{(V+E)\left(q_{i}-K_{i}\right)}}$.

Note that the reflection coefficient is no longer a constant but depends on the specific values of $\alpha_{i}$ and $\theta_{i-1}$ associated with a particular reflection. Although the absolute value of the reflection coefficient is still one, it introduces a nontrivial phase, namely, $2 \omega_{i}+\delta_{i}$ for each reflection, which contributes to the phase accumulation in the quantization condition of the scarring states. For a billiard system confined by hard walls, i.e., 
$V \rightarrow \infty$, the parameters are $\lambda_{i}=1$ and $C=\sqrt{\left(E-m c^{2}\right) / E}$. According to Eq. (6), the attenuation coefficient $q_{i}$ is infinite, so the wave function outside the domain decays to zero for arbitrarily small distance from the boundary; i.e., the particle is confined within the billiard.

The phase $\delta_{i}$ is due to spin rotation and can be regarded [61] as a spin following a nonadiabatically changing magnetic field pointing to the same direction as $\mathbf{k}$. The quantity $\delta_{i}$ is thus the Berry geometric phase due to the cycling rotation of the spin. The dynamical phase $2 \omega_{i}$ can be related to the Pancharatnam phase [62], i.e., the solid angle $P_{I \bar{T} R}$ spanned by the incident wave $(I)$, the reflected wave $(R)$, and the direction perpendicular to transitive spinor wave function $(\bar{T})$ in the Poincaré sphere at the reflection point. Thus, the quantity $2 \omega_{i}$ also has a geometrical origin.

To be specific, it is useful to describe spin polarization using the Bloch sphere, on which each point is an eigenstate $\chi_{i}, i=I, R, \bar{T}$ of the spin polarization matrix:

$$
S(\mathbf{r})=\frac{\hbar}{2}\left(\begin{array}{cc}
z & x-i y \\
x+i y & -z
\end{array}\right)=\frac{\hbar}{2}\left(\begin{array}{cc}
\cos \Theta_{r} & \sin \Theta_{r} e^{-i \phi} \\
\sin \Theta_{r} e^{i \phi} & -\cos \Theta_{r}
\end{array}\right),
$$

where $\cos \Theta_{r}=m c^{2} / E$. The argument of the reflection coefficient is given by

$$
\arg (R)=\arg \left(-\frac{\chi_{\bar{T}}^{\dagger} \chi_{I}}{\chi_{\bar{T}}^{\dagger} \chi_{R}}\right)=\pi+P_{I \bar{T} R}-\arg \left(\chi_{I}^{\dagger} \chi_{R}\right),
$$

where

$$
\begin{aligned}
& \left|\chi_{I}\right\rangle=\left(\begin{array}{c}
\cos \frac{\Theta_{r}}{2} e^{-i \theta_{i-1} / 2} \\
\sin \frac{\Theta_{r}}{2} e^{i \theta_{i-1} / 2}
\end{array}\right), \quad\left|\chi_{R}\right\rangle=\left(\begin{array}{c}
\cos \frac{\Theta_{r}}{2} e^{-i \theta_{i} / 2} \\
\sin \frac{\Theta_{r}}{2} e^{i \theta_{i} / 2}
\end{array}\right), \\
& \left|\chi_{T}\right\rangle=\frac{1}{\sqrt{2}}\left(\begin{array}{c}
-i e^{-i \alpha_{i} / 2} \\
e^{i \alpha_{i} / 2}
\end{array}\right), \quad\left|\chi_{\bar{T}}\right\rangle=\frac{1}{\sqrt{2}}\left(\begin{array}{c}
e^{-i \alpha_{i} / 2} \\
-i e^{i \alpha_{i} / 2}
\end{array}\right) .
\end{aligned}
$$

Since

$$
\chi_{I}^{\dagger} \chi_{R}=e^{i\left(\theta_{i}-\theta_{i-1}\right) / 2}\left(1+\kappa^{2} e^{-i\left(\theta_{i}-\theta_{i-1}\right)}\right),
$$

we could separate out a mass-independent phase $\delta_{i}=\left(\theta_{i}-\right.$ $\left.\theta_{i-1}\right) / 2$ to get

$$
2 \omega_{i}=-\pi-P_{I \bar{T} R}+\arg \left(1+\kappa^{2} e^{-i\left(\theta_{i}-\theta_{i-1}\right)}\right) .
$$

However, such a decomposition does not simplify the calculation of the phases. We thus use the criterion of mass dependence to decompose and analyze the phases.

In the massless limit $m \rightarrow 0$ (or $\kappa \rightarrow 1$ ), we have

$$
\begin{aligned}
\tan \omega_{i} & =-\frac{\sin \left(\alpha_{i}-\theta_{i-1}\right)+1}{\cos \left(\alpha_{i}-\theta_{i-1}\right)} \\
& =-\frac{1-\cos \left(\alpha_{i}-\theta_{i-1}+\pi / 2\right)}{\sin \left(\alpha_{i}-\theta_{i-1}+\pi / 2\right)} \\
& =-\tan \frac{1}{2}\left(\alpha_{i}-\theta_{i-1}+\pi / 2\right) .
\end{aligned}
$$

When examining the reflection coefficient as a whole, we find that its phase is $2 \omega_{i}+\left(\alpha_{i}-\theta_{i-1}+\pi / 2\right)=0$, indicating $R=1$. In this case, the system effectively becomes a massless Dirac billiard and chirality emerges for periodic orbits with an odd number of bounces due to the $\pi$ phase difference

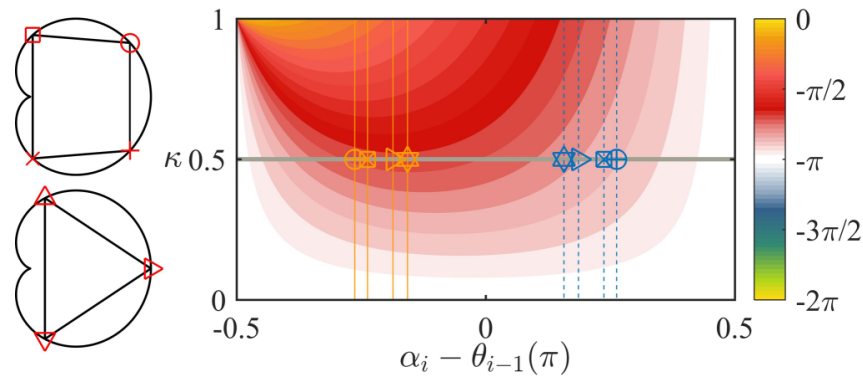

FIG. 3. Behaviors of the dynamical phase. Shown is the dynamical phase $\left(2 \omega_{i}\right)$ in the reflection coefficient for counterclockwise and clockwise orbits 3 and 4I. The symbols in the left panel mark the reflection points on the orbits. The same symbols on the right panel mark, for given $\kappa=0.5$, the value of $\alpha_{i}-\theta_{i-1}$ at the reflection points, with $\alpha_{i}-\theta_{i-1}<0(>0)$ for the counterclockwise (clockwise) case. The vertical lines mark the values of $\alpha_{i}-\theta_{i-1}$ for the reflection points.

associated with spin. For $m \rightarrow \infty(\kappa=0)$, the corresponding Dirac system degenerates to the Schrödinger system. In this limit, we have $\tan \omega_{i} \rightarrow-\infty$, and $2 \omega_{i} \rightarrow-\pi$. Thus, $e^{i 2 \omega_{i}} \rightarrow-1$, and the reflection coefficient becomes $R=-1$ in a Schrödinger billiard.

It is worth noting that, due to the uncertainty in the relative phase between the two components of the spinor wave function, while it is convenient to use symmetrical-plane wave solution (5) to solve the massive Dirac equation, there is flexibility in choosing the form of the two-component solution insofar as the ratio of the second component to the first is $e^{i \theta}$. However, this gauge freedom does not change the expression of the reflection coefficient and thus has no effects on the results.

\section{B. Dynamical phase and quantization condition of scarring states}

Define

$$
\beta^{ \pm}=\sum_{i=1}^{N} \delta_{i}^{ \pm}, \quad \gamma^{ \pm}=\sum_{i=1}^{N} 2 \omega_{i}^{ \pm},
$$

where $N$ is the number of reflections along the orbit and " \pm " denote the counterclockwise and clockwise orientations, respectively. Since $\delta_{i}^{ \pm}$(thus $\beta^{ \pm}$) depend only on the angles determined by the shape of the confinement boundaries and the classical periodic orbits, and persist for both massless and massive Dirac billiards, it is the primary phase. Besides its angle dependence, $2 \omega_{i}^{ \pm}$(hence $\gamma^{ \pm}$) also depends on the mass and wave number (or energy) through $\kappa$ [Eq. (2)]-it is thus a dynamical phase. Both phases have a geometrical origin.

From Eq. (9), we see that $\left(2 \omega_{i}^{ \pm}\right)$depends on both the angles $\alpha_{i}$ and $\theta_{i-1}$ and $\kappa$ that depends on the ratio of $k / m$ through Eq. (2). For a given scarring state, $k$ and $m$, and thus $\kappa$, are fixed. As a result, for a given periodic orbit as shown in Fig. 3, at each reflection point as marked by the symbols, the angles $\alpha_{i}$ and $\theta_{i-1}$ and consequently $\alpha_{i}-\theta_{i-1}$ can be determined. These are marked in the right panel of Fig. 3. The corresponding $\left(2 \omega_{i}^{ \pm}\right)$can then be calculated to yield $\gamma^{ \pm}$. Note that, for different orbits, these angles are different, and 
thus $\gamma^{ \pm}$will be different. Even for the same orbit but with a different orientation, the value of $\gamma^{ \pm}$can still be different. Another intriguing point is that, for different eigenstates, the wave numbers are different, so even for scarring states on the same orbit, the value of $\gamma^{ \pm}$will be different. Nevertheless, for a given system with fixed mass $m$, when the scarring orbit is also fixed, $\left(2 \omega_{i}^{ \pm}\right)$and thus $\gamma^{ \pm}$can be calculated as a function of the wave number $k$ or energy $E$.

We carry out a semiclassical analysis for the massive Dirac billiard system without a magnetic field. The phase accumulation about an orientated periodic orbit after traversing a complete cycle is

$$
\Phi^{ \pm}=\frac{1}{\hbar} S-\frac{\sigma \pi}{2}+\beta^{ \pm}+\beta^{ \pm}+\gamma^{ \pm}
$$

where $S=\int \mathbf{p} \cdot d \mathbf{r}=\hbar k L$ is the action, and $\sigma$ is the Maslov index that can be related to the number of conjugate points along the orbit, i.e., the number of reflections off the billiard boundary. The first $\beta^{ \pm}$in Eq. (11) is due to spin rotation during the reflection at the boundary, and $\beta^{ \pm}+\gamma^{ \pm}$is the nontrivial phase in the reflection coefficient. It is convenient to consider the two phases together and rewrite them as

$$
\Phi^{ \pm}=k L-\frac{\sigma \pi}{2}+\beta^{ \pm} \times 2+\gamma^{ \pm}
$$

The quantity $\beta^{ \pm}$can be calculated directly by following the orbits, which only has two values for typical periodic orbits: $\beta=0$ for orbits $3^{-}, 4 \mathrm{II}^{ \pm}, 5 \mathrm{I}^{+}$, and $5 \mathrm{II}^{+}$and $\beta=\pi \bmod$ $2 \pi$ for orbits $2^{ \pm}, 3^{+}, 4 \mathrm{I}^{ \pm}, 5 \mathrm{I}^{-}$, and $5 \mathrm{II}^{-}$(see Fig. 6 for the geometric shapes of these orbits). Since $\beta$ equals to either 0 or $\pi \bmod 2 \pi$, the quantity $\beta \times 2$ only contributes to integer multiples of $2 \pi$ to the total phase $\Phi$, which is independent of the mass, the energy, and the orbits. Thus, the key is the dynamical phase $\gamma^{ \pm}$.

For a scarring state to emerge, it is necessary that $\Phi^{ \pm}=$ $2 \pi n$ ( $n$ being an integer) be satisfied, which leads to the quantization condition for the wave number:

$$
k_{n}^{ \pm}=\left(2 \pi n+\sigma \pi / 2-2 \beta^{ \pm}-\gamma^{ \pm}\right) / L .
$$

Figure 4 plots $k_{n}^{ \pm}$versus $n$ for two representative scars on orbit 3 (upper left) and orbit 4I (lower right). The curves are theoretical predictions while the data points are eigenwave numbers $k_{n}^{ \pm}$for the scarring states identified manually through visual examination of all the available eigenstates. It can be seen that the prediction of the quantization formula agrees well with the numerical data. To see the fine variations of $\gamma^{ \pm}$ versus $k$, we define

$$
\Gamma^{ \pm}=\bmod \left(k_{n}^{ \pm} L, 2 \pi\right)=\bmod \left(\sigma \pi / 2-2 \beta^{ \pm}-\gamma^{ \pm}, 2 \pi\right) .
$$

The insets in Fig. 4 show $\bmod \left(k_{n}^{ \pm} L, 2 \pi\right)$ calculated directly from the scarring states (data points), together with the theoretical predictions $\bmod \left(\sigma \pi / 2-2 \beta^{ \pm}-\gamma^{ \pm}, 2 \pi\right)$ (curves). The two types of results are consistent with each other, revealing the effects of the variation of $\gamma^{ \pm}(k)$. For $k \rightarrow 0$, the system approaches the Schrödinger limit, and the difference in $\Gamma^{ \pm}$diminishes.

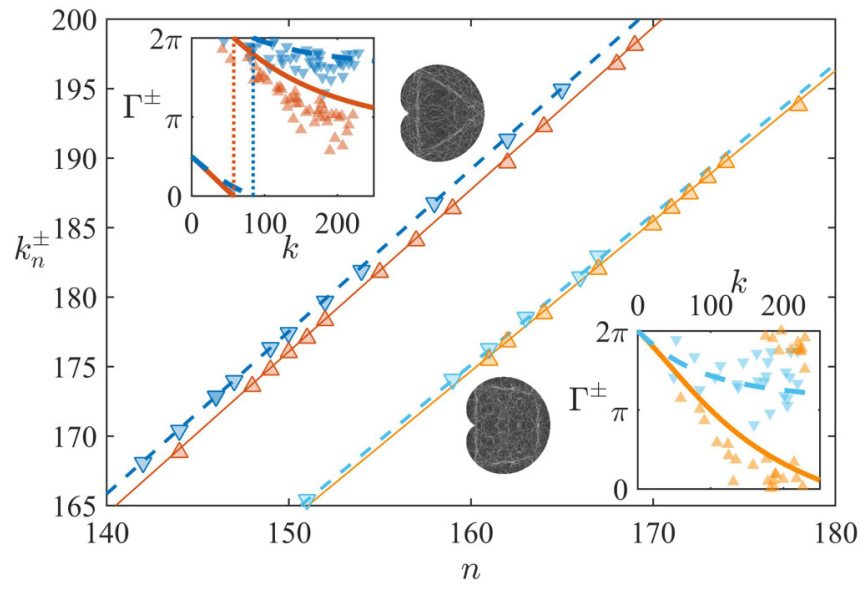

FIG. 4. Validity of the uncovered quantization formula. For the heart-shaped massive chaotic Dirac billiard, the semiclassical quantity $k_{n}^{ \pm}$versus $n$ from theory (curves) and the identified scarring states (symbols). The upper left inset is for orbit 3 (in darker color) and the lower right inset is for orbit $4 \mathrm{I}$ (in lighter color), where $\Gamma^{ \pm}$(see text) versus $k$ is shown. The mass is $m=100$. Orange upward- pointing triangles and solid curves are for scars with counterclockwise current orientation $(+)$, while blue downward-pointing triangles and dashed curve are for the clockwise orientation $(-)$.

\section{UNIFICATION OF NONRELATIVISTIC AND RELATIVISTIC SCARS}

\section{A. The effects of the dynamical phase}

Scars persist from $m=0$ to $m \rightarrow \infty$. As $m$ is increased from zero, how do scars transit from being relativistic chiral for $m=0$ to nonrelativistic quantum scars in the $m \rightarrow \infty$ limit? To address this fundamental issue in a concrete manner, we consider periodic orbits of odd periods (e.g., a triangular scar) because, for orbits of even periods (e.g., period 4), the corresponding scars are nonchiral in both the $m=0$ and $m \rightarrow$ $\infty$ limits. Does such a scar remain nonchiral for an arbitrary value of $m$ ? For a massless Dirac billiard, the characterization of chiral scars is that, for a given wave number, the difference $\Delta \Phi$ of the accumulated phase between counterclockwise and clockwise cycles is not an integer multiple of $2 \pi$. If the phase along one orientation satisfies the quantization condition, the other will not, breaking the time-reversal symmetry and leading to GUE (Gaussian unitary ensemble) level spacing statistics [49]. More specifically, in the massless case, we have $\Delta \Phi=\beta^{+}-\beta^{-}=\Delta \beta$. Since $\bmod (\Delta \beta, 2 \pi)$ equals $\pi$ for odd orbits and zero for even orbits, there will be a $\pi$ phase difference for periodic orbits with an odd number of bounces $[49,52,55]$. This is the fundamental mechanism for the subtle T-symmetry-breaking phenomenon in massless Dirac billiard systems.

For the massive Dirac billiard, since $\bmod (2 \Delta \beta, 2 \pi)=0$ holds regardless of whether the orbit is even or odd, the accumulated phase difference becomes $\Delta \Phi=\gamma^{+}-\gamma^{-}=$ $\Delta \gamma$. Thus, the key to understanding the transition from chiral scars to nonrelativistic scars is the dynamical phases $\gamma^{ \pm}$ (or $2 \omega_{i}^{ \pm}$) and their difference $\Delta \gamma$. Figure 5 shows, for two representative orbits, $\Delta \gamma$ versus $k$ and $m$. For orbit 3 , the range of $\Delta \gamma$ is $[0, \pi]$, while for orbit $4 \mathrm{I}$, the range is $[0,2 \pi]$. 

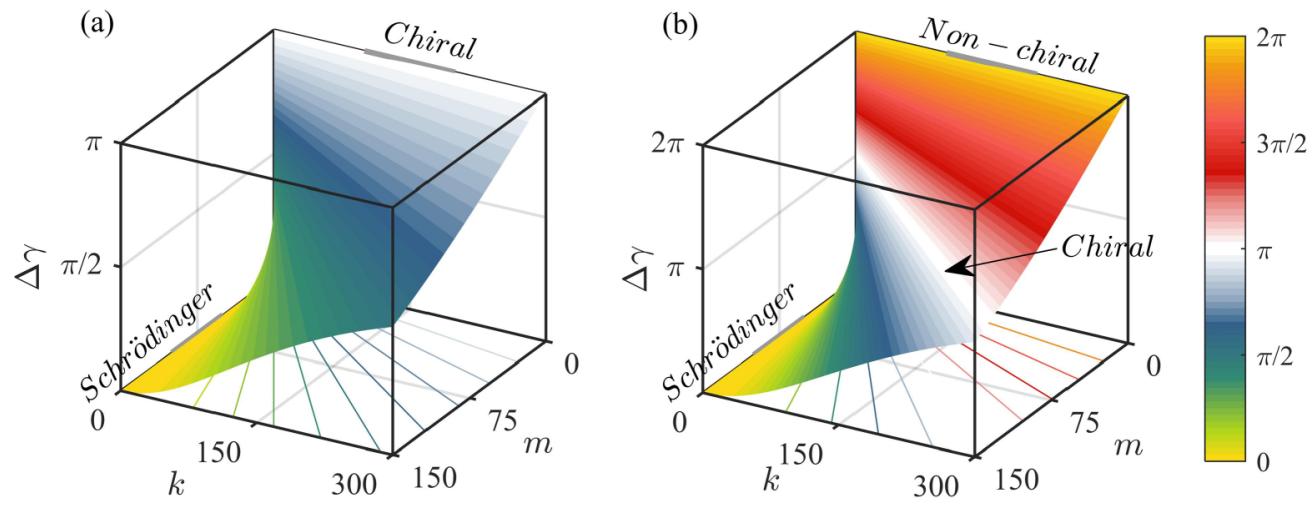

FIG. 5. Unification of relativistic and nonrelativistic quantum scars by the dynamical phase. Difference in the dynamical phase between counterclockwise and clockwise scars on orbits 3 (a) and 4I (b). The massless Dirac regime is $m \rightarrow 0$, while $k \rightarrow 0$ is the Schrödinger limit. Since $\kappa$ depends only on the ratio $k / m, \Delta \gamma$ takes the same values on the straight lines of $k \propto m$.

For both cases, the limit $k \rightarrow 0$ for a finite $m$ is equivalent to $m \rightarrow \infty$ for a fixed $k$ (the nonrelativistic limit) where $\Delta \gamma$ goes to zero. For $m \rightarrow 0$ (massless Dirac billiard), we have $\Delta \gamma \rightarrow \pi$ for orbit 3 , leading to chiral scars. For orbit 4I, we have $\Delta \gamma \rightarrow 2 \pi$, corresponding to nonchiral scars, which is consistent with previous observations [52,55]. Interestingly, for orbit 4I, around $k=2 m$, we have $\Delta \gamma \sim \pi$, exhibiting an accidental chiral nature.

\section{B. Understanding from length spectra analysis}

A convenient tool to reveal the signatures of classical periodic orbits is the length spectrum $[1,2,12]$. Denote $A^{ \pm}(L)$ as the contribution of states with counterclockwise-clockwise orientations of orbital length $L$. For a particular periodic orbit, we have

$$
A^{+}(L)=e^{i \Delta \Phi} A^{-}(L)=e^{i \Delta \gamma} A^{-}(L)
$$

for the massive Dirac billiard. As a result, the height of a peak in the length spectrum,

$$
\left|A^{+}(L)+A^{-}(L)\right|^{2}=\left|1+e^{i \Delta \gamma}\right|^{2}\left|A^{-}(L)\right|^{2},
$$

can be modulated by $\Delta \gamma$, as exemplified in Fig. 6(a), a series of spectra with increasing mass. We find that the mass has no effect on the orbits for which the current directions (i.e., counterclockwise or clockwise) cannot be distinguished, such as orbits 2 and 4II. However, for orbits whose current directions can be distinguished, regardless of whether they are even or odd, mass can affect the heights of the peaks in the spectrum. In particular, for odd orbits $(3,5 \mathrm{I}$, and 5II), for $m=0$, the $\pi$ phase difference in $\Delta \gamma$ makes the contributions to the spectrum from the counterclockwise and clockwise current states cancel each other exactly, so there are no peaks at the corresponding lengths, as evidenced in the bottom curve in Fig. 6(a). With increasing mass, the values of $\Delta \gamma$ for these orbits decrease from $\pi$ to zero, as shown in Fig. 6(b), leading to a continuous increase in the height of the peak at the corresponding length. For even orbits whose orientation can be distinguished, e.g., orbits 4I, 6I, and 6II, the height decreases first and then increases with the mass. This is because as the mass increases from zero to $\infty, \Delta \gamma$ decreases from $2 \pi$ to zero. Consequently, there exists an intermediate mass value (e.g., $m=100$ ) at which $\Delta \gamma \sim \pi$, where the peak height is greatly suppressed.
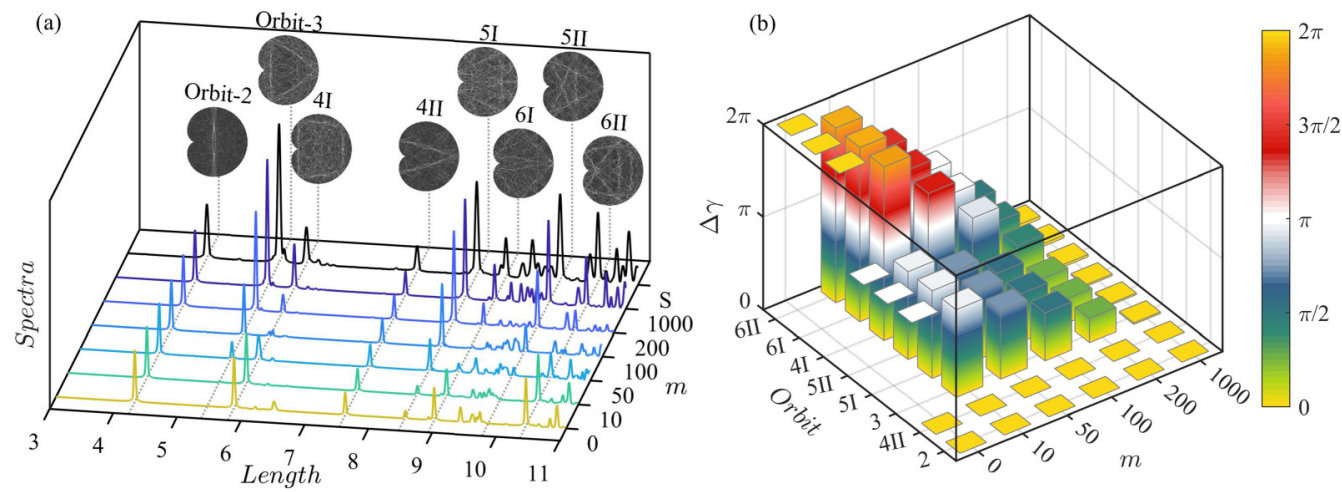

FIG. 6. Analysis of length spectra. (a) Length spectra for different masses: $m=0$ (massless Dirac billiard), 10, 50, 100, 200, 1000, and the Schrödinger limit. The peaks correspond to classical periodic orbits on which the scars are concentrated, as demonstrated in the insets. (b) $\Delta \gamma$ for these orbits vs $m$. Since $k \in\left(0, k_{\max }\right)$, where $k_{\max }$ is about $200, \Delta \gamma$ also takes values in a range, as marked by the solid bars. 
Additional results from length-spectra analysis for different energy ranges are presented in Appendix E.

\section{INDICATIONS OF T-SYMMETRY RESTORATION IN THE INFINITE MASS LIMIT}

Whether the system possesses $\mathrm{T}$ symmetry is largely determined by the dynamical phase. In the massless limit, we have $\Delta \gamma \rightarrow \pi$ for odd orbits, breaking $\mathrm{T}$ symmetry. For the massive case, $\Delta \gamma$ takes on finite values, so $\mathrm{T}$ symmetry is always broken but with a deteriorating effect as $m$ goes to infinity, where $\Delta \gamma \rightarrow 0$ holds for both odd and even orbits. As a result, $\mathrm{T}$ symmetry is restored so that scars with both orientations can occur simultaneously. This fundamental interplay between the dynamical phase and $\mathrm{T}$ symmetry has been verified through a systematic study of the level spacing statistics (e.g., for a fixed range of wave number) as the mass is increased from zero; see Sec. VA. Alternatively, this can be understood from the spin polarization on the boundary (see Sec. VB below). For a finite value of $m$, spin is polarized on the boundary due to the infinite mass confinement, breaking $\mathrm{T}$ symmetry. However, as $m$ goes to infinity, the expanding coefficients on the spin eigenstates go to zero continuously, leading to a zero wave function on the boundary in the limiting case, where $\mathrm{T}$ symmetry is restored.

\section{A. Statistics of energy level spacing for chaotic massive Dirac billiard systems}

Because of the fundamental role played by symmetry in quantum systems, we analyze two types of chaotic massive Dirac billiard systems: one with and another without a geometric symmetry, e.g., the heart-shaped and the Africa-shaped billiards.

For the heart-shaped chaotic massless Dirac billiard, although the system does not possess the time-reversal symmetry, it is invariant under the combined symmetric operation of time reversal and parity. As a result, the statistics of the energy level spacing are described by those of GOE [49]. In the infinite mass limit, the system degenerates to a nonrelativistic quantum billiard. In this case, due to the mirror symmetry, the eigenstates are either symmetric or antisymmetric, leading to two sets of independent eigenvalues. Consequently, the level spacing statistics follow those of double GOEs, i.e., a combination of the eigenlevels from two independent random matrices with each yielding GOE statistics. Figure 7 shows the level spacing statistics for three cases of different mass values, where the first 2800 levels are used to calculate the statistics. Indeed, for $m=0$, the statistics are GOE. For $m=1000$, the statistics of double GOEs arise. For $m=200$, the results are in between the two limiting cases.

For the Africa-shaped billiard that does not possess any geometric symmetry, in the massless limit, there is T-symmetry breaking. In this case, the spectral fluctuation properties of the energy levels are described by those of GUE [49]. However, in the large mass limit, GOE statistics emerge due to the restoration of $\mathrm{T}$ symmetry caused by the dynamical phase. These results are exemplified in Fig. 8.
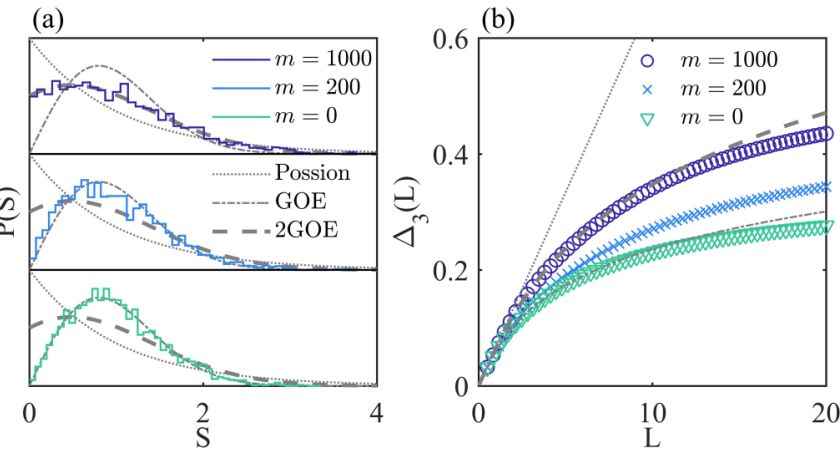

FIG. 7. Energy level spacing statistics for the heart-shaped massive Dirac billiard system. (a) Level spacing distribution $P(S)$ calculated from 2800 levels, where the three rows (from top to bottom) correspond to mass values $m=1000,200,0$, respectively. (b) The corresponding spectral rigidity $\Delta(L)$.

\section{B. Spin polarization on boundary}

To better understand the effect of domain boundary on spin, we analyze the influence of mass on the interaction between the spin degree of freedom and the boundary of a finite mass confinement. The spin operator in the $y$ direction, $\hat{S}_{y}=(\hbar / 2) \hat{\sigma}_{y}$, has two eigenfunctions:

$$
\hat{S}_{y}\left|\chi_{\uparrow \downarrow}\right\rangle= \pm \frac{\hbar}{2}\left|\chi_{\uparrow \downarrow}\right\rangle,
$$

where

$$
\left|\chi_{\uparrow}\right\rangle=\frac{1}{\sqrt{2}}\left(\begin{array}{l}
1 \\
i
\end{array}\right), \quad\left|\chi_{\downarrow}\right\rangle=\frac{1}{\sqrt{2}}\left(\begin{array}{c}
1 \\
-i
\end{array}\right) .
$$

For a massive wave function on the boundary given by

$$
\begin{aligned}
\psi & =\sqrt{\frac{E+m c^{2}}{2 E}}\left(\begin{array}{c}
e^{-i \theta_{i-1} / 2}+R_{i} e^{-i \theta_{i} / 2} \\
\kappa\left(e^{i \theta_{i-1} / 2}+R_{i} e^{i \theta_{i} / 2}\right)
\end{array}\right) \\
& =\sqrt{\frac{E+m c^{2}}{2 E}} 2 e^{i\left(\omega_{i}-\theta_{i-1} / 2\right)}\left(\begin{array}{c}
\cos \omega_{i} \\
-i \kappa \sin \left(\omega_{i}-\theta_{i-1}\right)
\end{array}\right),
\end{aligned}
$$
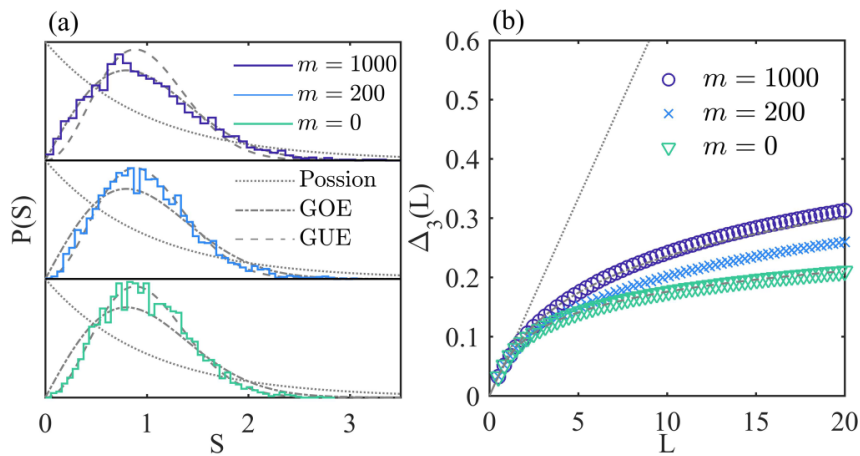

FIG. 8. Statistics of energy-level spacing for the chaotic Africashaped Dirac billiard system. Results from three cases are presented: the Schrödinger limit $(m=1000)$, massive Dirac $(m=200)$, and the massless Dirac $(m=0)$ from top to bottom, respectively. (a) Level spacing distributions $P(S)$ calculated from the first 3000 energy levels. (b) The spectral rigidity $\Delta(L)$ for the same set of energy levels. 
we can expand it in the eigenfunction base of $\hat{S}_{y}: \Psi=$ $\sum_{i=\uparrow \downarrow} A_{i}\left|\chi_{i}\right\rangle$, with the expansion coefficients given by

$$
\begin{aligned}
& A_{\uparrow}=\sqrt{\frac{E+m c^{2}}{E}} e^{i\left(\omega_{i}-\theta_{i-1} / 2\right)}\left[1-\kappa \frac{\sin \left(\omega_{i}-\theta_{i-1}\right)}{\cos \omega_{i}}\right] \cos \omega_{i}, \\
& A_{\downarrow}=\sqrt{\frac{E+m c^{2}}{E}} e^{i\left(\omega_{i}-\theta_{i-1} / 2\right)}\left[1+\kappa \frac{\sin \left(\omega_{i}-\theta_{i-1}\right)}{\cos \omega_{i}}\right] \cos \omega_{i} .
\end{aligned}
$$

With the simplification

$$
\begin{aligned}
\kappa \frac{\sin \left(\omega_{i}-\theta_{i-1}\right)}{\cos \omega_{i}} & =\kappa \tan \omega_{i} \cos \theta_{i-1}-\kappa \sin \theta_{i-1} \\
& =\kappa \cdot \frac{\frac{\kappa}{\lambda_{i}} \sin \theta_{i-1}-1}{\frac{\kappa}{\lambda_{i}} \cos \theta_{i-1}} \cdot \cos \theta_{i-1}-\kappa \sin \theta_{i-1} \\
& =\lambda_{i},
\end{aligned}
$$

we obtain

$$
\begin{aligned}
& A_{\uparrow}=\left(1+\lambda_{i}\right) \sqrt{\frac{E+m c^{2}}{E}} e^{i\left(\omega_{i}-\theta_{i-1} / 2\right)} \cos \omega_{i}, \\
& A_{\downarrow}=\left(1-\lambda_{i}\right) \sqrt{\frac{E+m c^{2}}{E}} e^{i\left(\omega_{i}-\theta_{i-1} / 2\right)} \cos \omega_{i} .
\end{aligned}
$$

The massive wave function is a superposition state of the eigenfunctions of $\hat{S}_{y}$. When the boundary is confined by an infinite mass, we have $\lambda_{i}=1$ and the expansion coefficients associated with the down $y$-spin eigenfunction are zero, regardless of the mass inside. That is, the wave function can be regarded as an up-spin eigenfunction of $\hat{S}_{y}: \psi=A_{\uparrow}\left|\chi_{\uparrow}\right\rangle$ and $\hat{S}_{y} \psi=\frac{\hbar}{2} \psi$.

It is useful to examine two limiting cases: $m=0$ and $m=\infty$. For the former case, the parameter values are $\kappa=1$ and $\omega_{i}=\theta_{i-1} / 2-\pi / 4$. The expansion coefficients are $A_{\uparrow}=$ $2 e^{-i \pi / 4} \cos \left(\theta_{i-1} / 2-\pi / 4\right)$ and $A_{\downarrow}=0$, which are consistent with the spinor wave function for massless Dirac fermions discussed in Ref. [55]. In the latter case (the infinite mass limit), the parameter values are $\kappa=0$ and $\omega_{i}=-\pi / 2$. We have $A_{\uparrow}=0$ and $A_{\downarrow}=0$, indicating that the wave function on the boundary is zero: the boundary condition in a nonrelativistic quantum billiard system.

To further validate the boundary effect on spin, we analyze the spatial distribution of the current density. The current operator $[49,55]$ is $\hat{\mathbf{u}}=\nabla_{\mathbf{p}} \hat{H}=c \hat{\boldsymbol{\sigma}}$ and the current density, i.e., the expectation value of the current operator, can be written as

$$
\mathbf{J}=c \Psi^{\dagger} \hat{\boldsymbol{\sigma}} \Psi=2 c\left[\operatorname{Re}\left(\psi_{1}^{*} \psi_{2}\right), \operatorname{Im}\left(\psi_{1}^{*} \psi_{2}\right)\right] .
$$

Inside and outside the billiard domain, the current expressions are, respectively,

$$
\begin{aligned}
& J_{x}^{\text {in }}=0, \\
& J_{y}^{\text {in }}=\frac{\hbar c^{2} k}{E}\left[2 \sin \theta_{i-1}+2 \sin \left(2 k x \cos \theta_{i-1}-2 \gamma_{n}+\theta_{i-1}\right)\right]
\end{aligned}
$$
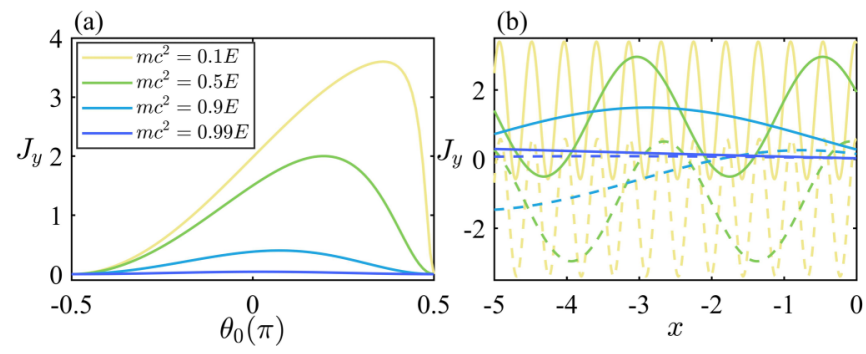

FIG. 9. Effect of mass on current density. (a) Dependence of the $y$ component of the boundary current on the incident angle $\theta_{i-1}$ for different values of the mass portion of the energy $m c^{2} / E$. (b) The $y$ component of the current inside the billiard domain vs the mass portion for a fixed incident angle. The solid and dashed curves represent the currents in the counterclockwise and clockwise directions, respectively. The mass conditions are specified in the inset.

and

$$
\begin{aligned}
& J_{x}^{\text {out }}=0, \\
& J_{y}^{\text {out }}=c|T|^{2} e^{-2 q x}=\frac{4 c\left(E-m c^{2}\right)}{E} \sin ^{2}\left(\gamma_{n}-\theta_{i-1}\right) e^{-2 q x} .
\end{aligned}
$$

The current normal to the boundary, $J_{x}$, is always zero because of Hermiticity of the Hamiltonian. Because of the infinite mass confinement $(q \rightarrow \infty)$, the decay factor $e^{-2 q x}$ in $J_{y}^{\text {out }}$ will decrease rapidly to zero. It then suffices to focus on the current component $J_{y}^{\text {in }}$. As illustrated in Fig. 9(a), the boundary current always points in the positive direction, independent of the incident angle. As the mass portion of the energy increases, the current decreases and tends to zero for large masses. Figure 9(b) shows that, for a fixed incident angle, the current inside exhibits a periodic behavior with an increasing period but its amplitude decreases with the mass. In the large mass regime, the current concentrates on the $x$ axis, i.e., $J_{y} \rightarrow 0$. These results agree with those from the analysis of the spin operator $\hat{S}_{y}$.

\section{DISCUSSION}

Scars, relatively high concentrations of the quantum wave functions along classical unstable periodic orbits, are perhaps the most fundamental manifestation of classical chaotic behavior in the corresponding quantum system, nonrelativistic or relativistic. In the traditional field of quantum chaos that deals with nonrelativistic quantum systems described by scalar wave functions governed by the Schrödinger equation, the phenomenon of scarring has been extensively studied in the past four decades [4,6-34,36]. In the recently emergent field of relativistic quantum chaos $[47,48]$ that treats systems described by spinor wave functions obeying the Dirac equation, there has also been a particular focus on scarring of massless Dirac fermions. In spite of the common feature between nonrelativistic and relativistic quantum scars, there are characteristic differences. In particular, for all scars in nonrelativistic quantum systems and most scarred states in massless relativistic quantum systems, the associated wave functions return completely to themselves after traversing the unstable 
periodic orbits once. That is, for such a scar, the phase change after one round trip along the unstable periodic orbit is zero or $2 \pi$. The most pronounced difference between scars in nonrelativistic and relativistic quantum systems of massless Dirac fermions uncovered so far is the emergence of a class of scars in the latter that have no counterparts in the former: Chiral scars that require two round trips along the unstable periodic orbits for the underlying spinor wave functions to return completely to themselves. The phase change associated with a chiral scar after traversing the classical periodic orbit once is thus $\pi$. In a hard wall billiard system, chiral scars arise on classical unstable periodic orbits with an odd number of bounces or reflections off the billiard boundary. In view of the characteristic difference, a question of fundamental interest is whether nonrelativistic and relativistic quantum scars are different entities, or are they just two different aspects of the same physics? In other words, does a theory exist unifying the scarring phenomena in nonrelativistic and relativistic quantum systems?

We have developed a theoretical framework to unify nonrelativistic and relativistic quantum scars. The basic idea is to investigate relativistic quantum chaotic systems of fermions governed by the massive Dirac equation. In the zero mass limit, the equation degenerates to the massless Dirac equation. In the large mass limit, the spinor components "decouple," e.g., the second component goes to zero, and the first component becomes one described by the Schrödinger equation with the Dirichlet boundary condition, degenerating effectively the system to a nonrelativistic quantum billiard. Scarring of massive Dirac fermions can thus serve as a bridge to unify nonrelativistic quantum and relativistic chiral scars. Especially when the mass is finite, the reflection coefficient at the boundary is no longer a constant but introduces a new phase that needs to be included in the quantization condition for scarring states. The difference in the dynamical phase, $\Delta \gamma$, which depends on the mass and wave number, plays a determining role. As the mass is increased from zero, $\Delta \gamma$ decreases from $\pi(2 \pi)$ for odd (even) orbits. For $m \rightarrow \infty$, one has $\Delta \gamma \rightarrow 0$, so that the orientation can be distinguished, leading to conventional scars in the nonrelativistic quantum billiard system, where T symmetry is restored, as corroborated by the level spacing statistics and the deterioration of the spin polarization effects on the boundary.

From the point of view of symmetry, whether the system possesses the $\mathrm{T}$ symmetry is largely determined by the dynamical, mass-dependent phase. In the large mass (or low kinetic energy) limit, this mass-dependent phase tends to zero, conserving $\mathrm{T}$ symmetry. As the mass portion of the total energy is reduced continuously, the dynamical phase becomes more dominant, breaking $\mathrm{T}$ symmetry. This fundamental interplay between the dynamical phase and $\mathrm{T}$ symmetry has been verified through a systematic study of the statistics of energy level spacing in the massive Dirac billiard systems in general and in the limiting cases of zero and infinite mass.

\section{ACKNOWLEDGMENTS}

This work was supported by NNSF of China under Grants No. 11775101 and No. 11422541. H.Y.X. and Y.C.L. are supported by the Pentagon Vannevar Bush Faculty Fellowship program sponsored by the Basic Research Office of the Assistant Secretary of Defense for Research and Engineering and funded by the Office of Naval Research through Grant No. N00014-16-1-2828.

\section{APPENDIX A: DEGENERACY OF MASSIVE DIRAC BILLIARD SYSTEM INTO A NONRELATIVISTIC QUANTUM BILLIARD IN THE INFINITE MASS LIMIT}

In the infinite mass limit, the massive Dirac billiard system degenerates into the corresponding nonrelativistic quantum billiard, which can be seen as follows: Consider a twodimensional spin-1/2 particle of mass $m$ confined by an infinite mass potential in a billiard domain $\mathcal{D}$. In the position representation, the Dirac Hamiltonian $\hat{H}$ acts on the twocomponent spinor wave function $\psi(\mathbf{r})=\left[\psi_{1}(\mathbf{r}), \psi_{2}(\mathbf{r})\right]^{\mathrm{T}}$. The massive Dirac equation is given by Eq. (1). The confinement potential is given by

$$
V(\mathbf{r})= \begin{cases}0, & \text { inside } \mathcal{D}, \\ \infty, & \text { outside } \mathcal{D} .\end{cases}
$$

Hermiticity of the Hamiltonian in Eq. (1) requires that the outward current vanish, which is equivalent to the condition: $\psi_{2} / \psi_{1}=i B \exp \{i \alpha\}$, where $B$ is a constant. The infinite mass confinement leads to $B=1$, ensuring that the boundary condition of the massive Dirac billiard is identical to its massless counterpart:

$$
\frac{\psi_{2}}{\psi_{1}}=i \exp \{i \alpha\}
$$

where $\alpha$ is the angle of the normal vector of the boundary measured from the positive horizontal direction.

When the kinetic energy is small compared with the static energy, a Taylor expansion of the energy expression gives $E=$ $\sqrt{(\hbar c k)^{2}+\left(m c^{2}\right)^{2}} \approx m c^{2}+\hbar^{2} k^{2} /(2 m)$. The zeroth-order approximation of $\psi(\mathbf{r}, t)$ can be written as

$$
\psi(\mathbf{r}, t)=\psi(\mathbf{r}) e^{-i E t / \hbar} \approx \psi(\mathbf{r}) e^{-i m c^{2} t / \hbar},
$$

which is a solution of

$$
i \hbar \frac{\partial \psi(\mathbf{r}, t)}{\partial t} \approx m c^{2} \psi(\mathbf{r}, t) .
$$

In the polar coordinates $\mathbf{r}=(r, \phi)$, the Dirac equation

$$
i \hbar \frac{\partial \psi_{1}(\mathbf{r}, t)}{\partial t}=\hat{H} \psi_{1}(\mathbf{r}, t)
$$

in $\mathcal{D}$ can be expanded as

$$
\begin{aligned}
i \hbar \frac{\partial \psi_{1}(\mathbf{r}, t)}{\partial t} & =-i \hbar c e^{-i \phi}\left(\partial_{r}-\frac{i}{r} \partial_{\phi}\right) \psi_{2}(\mathbf{r}, t)+m c^{2} \psi_{1}(\mathbf{r}, t), \\
i \hbar \frac{\partial \psi_{2}(\mathbf{r}, t)}{\partial t} & =-i \hbar c e^{i \phi}\left(\partial_{r}+\frac{i}{r} \partial_{\phi}\right) \psi_{1}(\mathbf{r}, t)-m c^{2} \psi_{2}(\mathbf{r}, t) .
\end{aligned}
$$

Using the second component of Eq. (A2) in Eq. (A4b), we obtain the expression for $\psi_{2}(\mathbf{r}, t)$ in terms of $\psi_{1}(\mathbf{r}, t)$ :

$$
\psi_{2}(\mathbf{r}, t)=-\frac{i \hbar}{2 m c} e^{i \phi}\left(\partial_{r}+\frac{i}{r} \partial_{\phi}\right) \psi_{1}(\mathbf{r}, t) .
$$


Substituting Eq. (A5) into Eq. (A4a) yields the equation for the first component $\psi_{1}(\mathbf{r}, t)$ :

$$
i \hbar \frac{\partial \psi_{1}(\mathbf{r}, t)}{\partial t}=-\frac{\hbar^{2}}{2 m} \nabla^{2} \psi_{1}(\mathbf{r}, t)+m c^{2} \psi_{1}(\mathbf{r}, t) .
$$

Since $i \hbar \partial \psi_{1}(\mathbf{r}, t) / \partial t=E \psi_{1}$ and $E \approx m c^{2}+\hbar^{2} k^{2} /(2 m)$, combining with Eq. (A6), we have

$$
\nabla^{2} \psi_{1}(\mathbf{r})+k^{2} \psi_{1}(\mathbf{r})=0,
$$

which is the Helmholtz equation, i.e., the spatial part of the Schrödinger equation for a quantum billiard system. From Eq. (A5), we see that, as $m \rightarrow \infty, \psi_{2} \rightarrow 0$ holds uniformly inside the billiard. The boundary condition (A1) can be rewritten as $\psi_{2}=\psi_{1} i \exp (i \alpha)$. Since $\psi_{2} \rightarrow 0$, the boundary condition for $\psi_{1}$ becomes $\psi_{1}=0$.

We thus have that, in the $m \rightarrow \infty$ limit, the massive Dirac billiard degenerates to a nonrelativistic quantum system: $\psi_{2}=$ 0 , and the first component $\psi_{1}$ is governed by the Schrödinger equation (Helmholtz equation) with the Dirichlet boundary condition.

\section{APPENDIX B: ANALYTICAL SOLUTIONS OF CIRCULAR MASSIVE DIRAC BILLIARD}

The massive Dirac equation [Eq. (1)] in the polar coordinates $\mathbf{r}=(r, \phi)$ is

$$
\begin{aligned}
& m c^{2} \psi_{1}-i \hbar c e^{-i \phi}\left(\frac{\partial}{\partial r}-\frac{i \partial}{r \partial \phi}\right) \psi_{2}=E \psi_{1} \\
& -i \hbar c e^{i \phi}\left(\frac{\partial}{\partial r}+\frac{i \partial}{r \partial \phi}\right) \psi_{1}-m c^{2} \psi_{2}=E \psi_{2} .
\end{aligned}
$$

Eliminating $\psi_{1}$, we get the Legendre equation

$$
\frac{(\hbar c)^{2}}{m c^{2}-E}\left[\frac{\partial^{2}}{\partial r^{2}}+\frac{\partial}{r \partial r}+\frac{\partial^{2}}{r^{2} \partial \phi^{2}}\right] \psi_{2}=\left(m c^{2}+E\right) \psi_{2} .
$$

Using separation of variables, $\psi_{2}=R(r) \Phi(\phi)$, we obtain the analytical solution in the form of the Bessel functions:

$$
\psi_{2}=C_{2} J_{l}(k r) e^{i l \phi} .
$$

Substituting this into Eq. (B1) and using the recurrence relation of the Bessel functions: $v J_{v}(x)+x J_{v}^{\prime}(x)=x J_{v-1}(x)$, we get

$$
\psi_{1}=\frac{i C_{2} \hbar c k}{m c^{2}-E} J_{l-1}(k r) e^{i(l-1) \phi} .
$$

Setting $C_{1}=\frac{i C_{2} \hbar c k}{m c^{2}-E}$, we can rewrite the spinor wave function as

$$
\psi(\mathbf{r})=\left(\begin{array}{c}
C_{1} J_{l}(k r) e^{i l \phi} \\
C_{2} J_{l+1}(k r) e^{i(l+1) \phi}
\end{array}\right) .
$$

Taking into account the boundary condition $\psi_{2} / \psi_{1}=i e^{i \phi}$, we have

$$
\frac{C_{2}}{C_{1}} \frac{J_{l+1}(k r)}{J_{l}(k r)} e^{i \phi}=i \kappa \frac{J_{l+1}(k r)}{J_{l}(k r)} e^{i \phi}=i e^{i \phi},
$$

which yields the following equation for solving the eigenwave number,

$$
J_{l}(k r)=\kappa J_{l+1}(k r)
$$
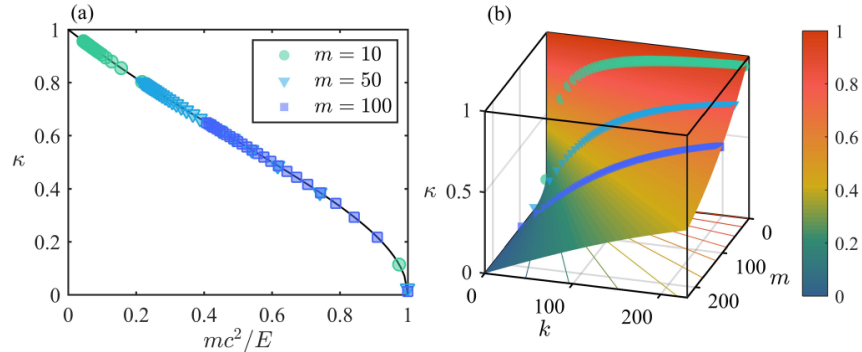

FIG. 10. Dependence of the key quantity $\kappa$ on mass and wave vector in the massive Dirac billiard. Shown is the dependence of $\kappa$ on the mass portion of energy (a) and on both mass $m$ and wave vector $k$ (b). The symbols are the analytical solutions for circular massive Dirac billiards with $m=10,50,100$. The contour lines show that $\kappa$ is invariant when $k$ is varied linearly with $m$, which can also be seen from Eq. (2) as $\kappa$ depends only on the ratio of $k / m$.

where $\kappa$ is given by Eq. (2), which plays a determining role in the dynamical phase and whose behavior is shown in Fig. 10. The eigenvalues $k_{l m}$ of the circular massive Dirac billiard can be obtained from Eq. (B4), and the eigenwave function is

$$
\psi(\mathbf{r})=N_{l m} e^{i l \phi}\left(\begin{array}{c}
J_{l}\left(k_{l m} r\right) \\
i \kappa_{l m} J_{l+1}\left(k_{l m} r\right) e^{i \phi}
\end{array}\right),
$$

with the normalization factor

$$
N_{l m}=\left[2 \pi \int_{0}^{1} d r r\left(J_{l}^{2}\left(k_{l m} r\right)+\kappa_{l m}^{2} J_{l+1}^{2}\left(k_{l m} r\right)\right)\right]^{-1 / 2} .
$$

Under the operation of the antiunitary operator $\hat{A}=\hat{\sigma}_{x} \hat{K}$, the Hamiltonian becomes $\hat{H}^{\prime}=\hat{A} \hat{H} \hat{A}^{-1}=-\hat{H}$, thus the eigenvalue is $E^{\prime}=-E$, and the corresponding eigenstate becomes

$$
\Psi^{\prime}=\hat{A} \Psi=\left(\begin{array}{c}
\psi_{2}^{*} \\
\psi_{1}^{*}
\end{array}\right)=N_{l m} e^{-i l \phi}\left(\begin{array}{c}
-i \kappa_{l m} J_{l+1}\left(k_{l m} r\right) e^{-i \phi} \\
J_{l}\left(k_{l m} r\right)
\end{array}\right) .
$$

\section{APPENDIX C: GENERALIZED CONFORMAL MAPPING METHOD FOR CHAOTIC MASSIVE DIRAC BILLIARDS}

For a massless chaotic Dirac billiard, the method of conformal mapping is effective, which enables a large number of eigenvalues and eigenstates to be calculated accurately and efficiently. The starting point to generalize this method to massive Dirac billiard systems is the eigenfunctions $\psi_{l m}(\mathbf{r})$ of a circular massive Dirac billiard system, which can be written down analytically (Sec. B):

$$
\begin{aligned}
\psi_{l m}(\mathbf{r}) & =N_{l m} e^{i l \phi}\left(\begin{array}{c}
J_{l}\left(\mu_{l m} r\right) \\
i \kappa_{l m} J_{l+1}\left(\mu_{l m} r\right) e^{i \phi}
\end{array}\right), \\
N_{l m} & =\left[2 \pi \int_{0}^{1} d r r\left(J_{l}^{2}\left(\mu_{l m} r\right)+\kappa_{l m}^{2} J_{l+1}^{2}\left(\mu_{l m} r\right)\right)\right]^{-1 / 2},
\end{aligned}
$$

where $\mu_{l m}$ is the eigenwave vector given by the equation $J_{l}\left(\mu_{l m}\right)=\kappa_{l m} J_{l+1}\left(\mu_{l m}\right)$, and $l$ and $m$ are the radial and angular subindices, indicating that the corresponding values should be calculated using the eigenvalues.

For a chaotic billiard with an analytic boundary in the $w=$ $(u, v)$ plane such as the heart-shaped or the Africa-shaped 
billiard, the domain can be transformed from a circle in the $z=(x, y)=(r, \phi)$ plane through the conformal mapping:

$$
u(x, y)+i v(x, y)=w(z)=\frac{z+c_{1} z^{2}+c_{2} e^{i d} z^{3}}{\sqrt{1+2 c_{1}^{2}+3 c_{2}^{2}}},
$$

where, for the heart-shaped and Africa-shaped billiards, the parameters are $\left(c_{1}=0.49, c_{2}=d=0\right)$ and $\left(c_{1}=c_{2}=\right.$ $0.2, d=\pi / 3$ ), respectively. When being acted upon by the Hamiltonian operator, since the Pauli matrices are anticommutative, the Dirac equation (1) inside the billiard region in the $(u, v)$ coordinates becomes

$$
\left[-\hbar^{2} c^{2} \Delta_{u v}+m^{2} c^{4}\right] \Psi=E^{2} \Psi
$$

or

$$
-\Delta_{u v} \Psi=k^{2} \Psi \text {. }
$$

The wave functions in the $w$ and $z$ planes are approximately equal, i.e., $\Psi(w)=e^{i \sigma_{z} \theta_{\mathrm{cm}} / 2} \Psi^{\prime}(x, y) \approx \Psi^{\prime}(z)$, where $\theta_{\mathrm{cm}}=$ $\arg (d w / d z)$, due to the rotation of the spinor wave function caused by the conformal mapping. To solve Eq. (C3), one needs to transform back from the chaotic billiard domain in the $w$ plane to the circular domain in the $z$ plane. With

$$
\Delta_{r \phi}=\left|\frac{d w}{d z}\right|^{2} \Delta_{u v} \equiv T(r, \phi) \Delta_{u v},
$$

Eq. (C3) becomes

$$
-\Delta_{r \phi} \Psi^{\prime}=k^{2} T(r, \phi) \Psi^{\prime} .
$$

Thus, as the billiard domain becomes regular, the equation becomes complex due to the quantity $T(r, \phi)$. Nevertheless, the eigensolutions Eq. (C1) for the circular massive Dirac billiard [for which $T(r, \phi)=1$ ] form an orthonormal basis for the general spinor wave functions satisfying the boundary condition (A1). Expanding $\Psi^{\prime}$ in this basis yields

$$
\Psi^{\prime}=\sum_{l^{\prime} m^{\prime}} C_{l^{\prime} m^{\prime}} \psi_{l^{\prime} m^{\prime}}(r, \phi) \text {. }
$$

Practically, the summation in Eq. (C5) needs to be truncated at a large but finite index. In our calculation, we choose the first 40000 eigenstates in the increasing order of the eigenwave number $\mu_{l m}$. Substituting this expansion into Eq. (C4) and carrying out the inner product with $\left\langle\psi_{l m}\right|$ on both sides, we obtain the following eigenequation:

$$
\sum_{l^{\prime} m^{\prime}} M_{l m l^{\prime} m^{\prime}} V_{l^{\prime} m^{\prime}}=V_{l m} / k^{2},
$$

where $V_{l m}=C_{l m} \mu_{l m}$ and

$$
\begin{aligned}
M_{l m l^{\prime} m^{\prime}}= & \frac{N_{l m} N_{l^{\prime} m^{\prime}}}{\mu_{l m} \mu_{l^{\prime} m^{\prime}}} \int_{0}^{2 \pi} e^{i\left(l^{\prime}-l\right) \phi} T(r, \phi) d \phi \\
& \times \int_{0}^{1} d r \cdot r\left[J_{l}\left(\mu_{l m} r\right) J_{l^{\prime}}\left(\mu_{l^{\prime} m^{\prime}} r\right)\right. \\
& \left.+\kappa_{l m} \kappa_{l^{\prime} m^{\prime}} J_{l+1}\left(\mu_{l m} r\right) J_{l^{\prime}+1}\left(\mu_{l^{\prime} m^{\prime}} r\right)\right] .
\end{aligned}
$$

From the eigenvalues $\lambda_{n}$ and the eigenvectors $V_{n}$ of the eigenequation $M V_{n}=\lambda_{n} V_{n}$, we can obtain the solutions of the massive Dirac equation with

$$
k_{n}=\sqrt{1 / \lambda_{n}}, \quad C_{n, l m}=V_{n, l m} / \mu_{l m} .
$$

The corresponding eigenenergy and eigenwave function are given by $E_{n}=\sqrt{\left(\hbar c k_{n}\right)^{2}+\left(m c^{2}\right)^{2}}$ and $\Psi_{n}^{\prime}(x, y)=$ $\sum_{l m} C_{n, l m} \psi_{l m}$ in the $(x, y)$ plane, respectively. Accordingly, in the $(u, v)$ plane, the eigenwave function is $\Psi_{n}(u, v)=$ $e^{i \sigma_{z} \theta_{\mathrm{cm}} / 2} \Psi_{n}^{\prime}(x, y)$.

Note that, due to the term $e^{i \sigma_{z} \theta_{\mathrm{cm}} / 2}$, the boundary condition for the massive Dirac billiard in the $(u, v)$ plane will not be transformed to the boundary condition of the same form as Eq. (A1) in the $(x, y)$ plane, especially at the boundary point where the value of $\theta_{\mathrm{cm}}$ is large. We have compared the results using the boundary integral and the conformal mapping methods for the heart-shaped billiard. There are noticeable discrepancies for the first few eigenstates in both the eigenwave numbers and eigenwave functions, where the wave functions are mostly localized on the boundaries. For large values of $n$, the discrepancies diminish.

The generalized conformal mapping method can yield a huge number of eigenwave functions simultaneously with high spatial resolution for the chaotic massive Dirac billiards, leading to a large number of scarring states for statistics and semiclassical analyses.

\section{APPENDIX D: ADDITIONAL RESULTS OF DYNAMICAL PHASE AND QUANTIZATION CONDITION OF SCARRING STATES}

The key to understanding the transition from chiral scars to nonrelativistic scars thus lies in the dynamical phase $\gamma^{ \pm}=$ $\sum_{i} 2 \omega_{i}^{ \pm}$. Examples of several orbits for $m=100$ are shown in Fig. 11, where both $\gamma^{+}, \gamma^{-}$and $\Delta \gamma=\gamma^{+}-\gamma^{-}$are plotted. It can be seen that $\gamma^{+}$and $\gamma^{-}$are different for all the orbits, and $\Delta \gamma$, the key to determining the chirality of the scars, exhibits different behaviors for even or odd orbits. As shown in the second row of Fig. 11, in the large- $k$ limit, the system approaches a massless Dirac billiard. In this case, for even orbits (e.g., type 4I), we have $\Delta \gamma \rightarrow 2 \pi$, while for odd orbits (types $3,5 \mathrm{I}$, and 5II), $\Delta \gamma \rightarrow \pi$ holds. This breaks the time-reversal symmetry because the $\pi$ phase difference renders impossible simultaneous quantization of the wave functions along the orbits in both orientations, in agreement with previous understandings of chiral scars in massless Dirac billiards [55].

For $k \rightarrow 0$, the system approaches the nonrelativistic limit. In this case, $\Delta \gamma$ goes to zero for all cases, indistinguishable for even and odd orbits. In between, $\Delta \gamma$ varies smoothly with $k$. However, as for even orbits, $\Delta \gamma$ changes from $2 \pi$ to 0 continuously, there will be a particular value $k$ (depending on the orbit) for which $\Delta \gamma=\pi$ holds. As a result, even orbits may also exhibit chirality. The change in $\Delta \gamma$ versus $k$ and the time-reversal symmetry breaking effect can be visualized through level spacing statistics (in Sec. V A).

The theoretical values of $\Gamma$ for a set of other periodic orbits together with those derived from the eigenwave numbers of the scarring states are plotted in Fig. 12. It can be seen that, despite fluctuations, the data follow the theoretical curves well. Period-2 and period-4-II orbits are bouncing-ball-like orbits. For such an orbit, itself and its time-reversed counterpart are identical, so no orientation can be defined. For period-5-I and period-5-II orbits, the orientations can be defined; thus, $\Gamma$ takes on different values for different orientations. For $k \rightarrow 0$, 
(a)

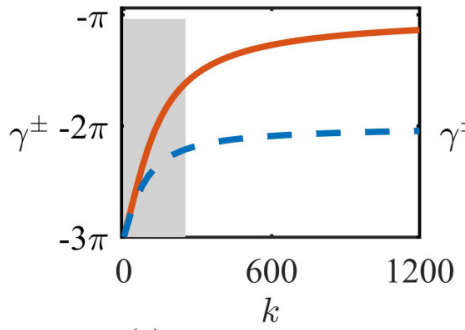

(e)

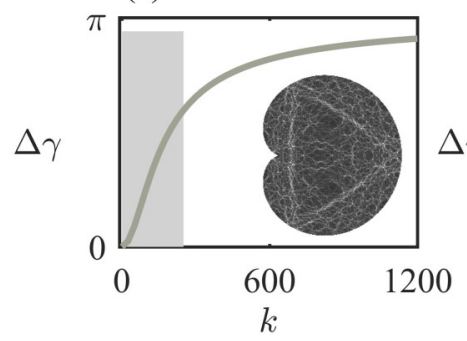

(b)

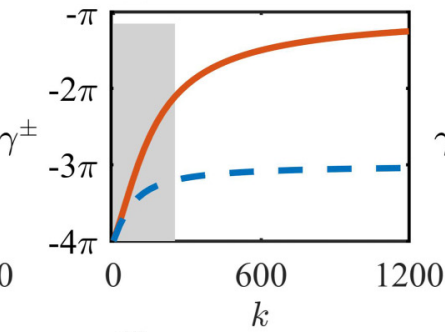

(f)

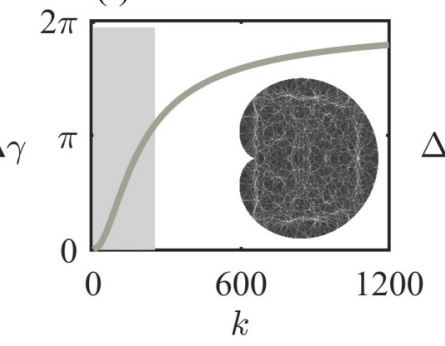

(c)

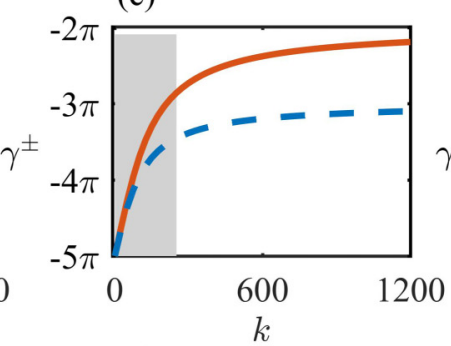

(g)
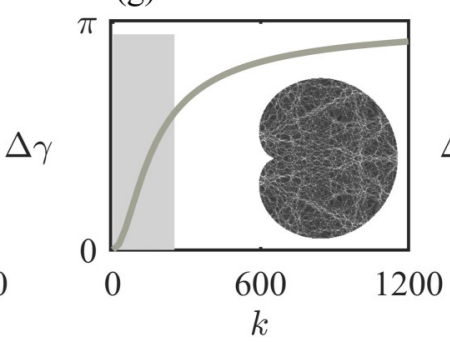

(d)

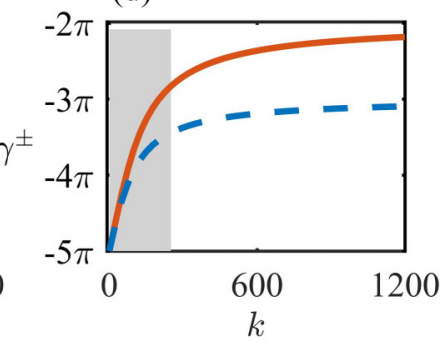

(h)

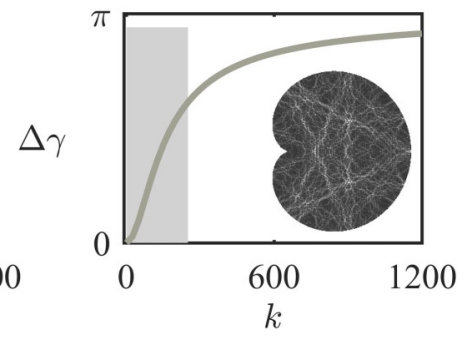

FIG. 11. Behaviors of the dynamical phase $\gamma=\sum_{i} 2 \omega_{i}$. From left to right are the phases for scars of types 3, 4I, 5I, and 5II, respectively, in the heart-shaped chaotic massive Dirac billiard. The mass is $m=100$. The solid orange and dashed blue curves in the first row [(a)-(d)] represent the theoretical values of the phases for counterclockwise and clockwise orbits. The range of the wave vectors is from 0 to 1200 . The second row [(e)-(h)] shows the phase difference $\Delta \gamma$ between the two cases and the gray-shaded areas represent the range of the wave numbers obtained numerically.

the system approaches the infinite mass limit, so the difference in $\Gamma$ diminishes. Since $\Gamma$ is the modular value of the phases, when it decreases to values below zero, we add a shift of $+2 \pi$,
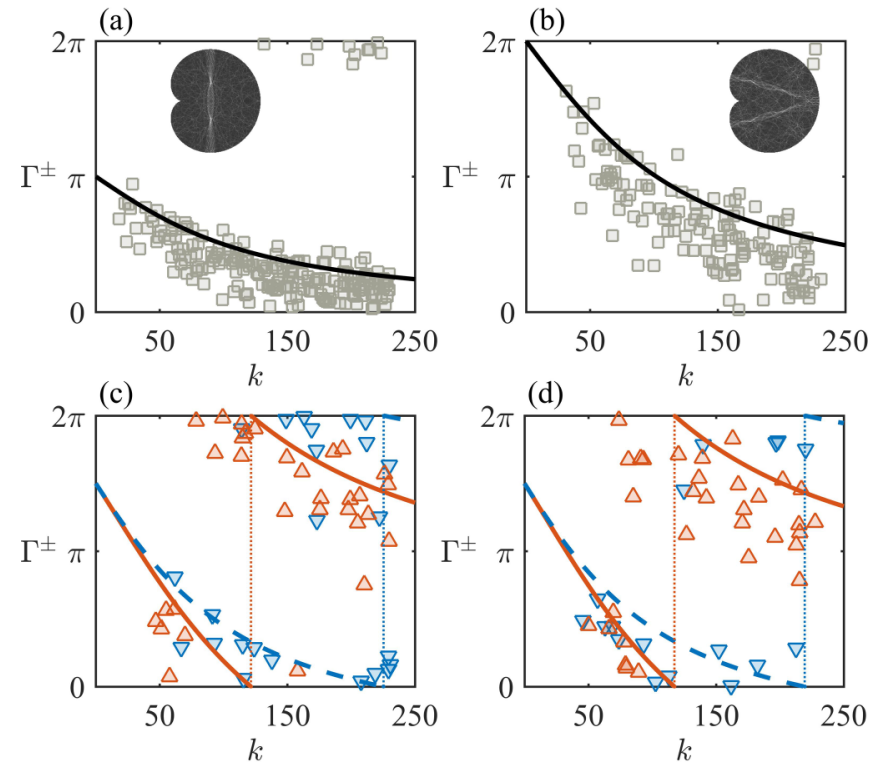

FIG. 12. $\Gamma$ characteristic for scars on different orbits for the heart-shaped massive Dirac billiard. The mass is $m=100$. [(a)-(d)] The $\Gamma$ values for scars of periods 2, 4-II, 5-I, and 5-II, respectively. The symbols are calculated via $\bmod (k L, 2 \pi)$. The curves are the theoretical predictions through $\gamma(m, k)$. Orange upward-pointing triangles and solid curve are for scars with counterclockwise current orientations, and blue downward-pointing triangles and dashed curve are for scars with clockwise orientations. Gray squares and black solid curve are for scars whose current directions cannot be distinguished. corresponding to the behavior of its decreasing from one in the $\Gamma$ plot.

Since only $\gamma$ depends on $k$, it is convenient to "normalize" $\Gamma$ as $\tilde{\Gamma}=\bmod (k L+\beta+\gamma, 2 \pi)=\bmod (\sigma \pi / 2-$ $\beta, 2 \pi)$. Again, $\tilde{\Gamma}$ can be calculated from eigenwave numbers $k$ of the scarring states through $\bmod (k L+\beta+\gamma, 2 \pi)$ and
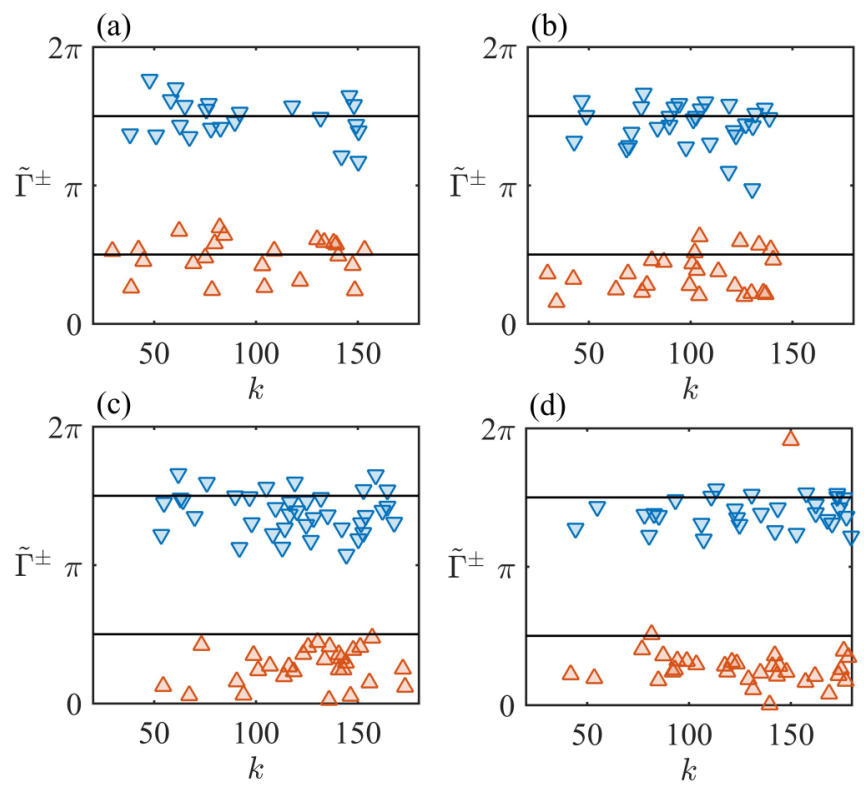

FIG. 13. Normalized values $\tilde{\Gamma}$ for period-3 scars at different mass values. Shown are the $\tilde{\Gamma}$ values for period-3 scars in the heart-shaped billiard system for $m=0,10,50,100$ [(a)-(d), respectively]. The horizontal solid lines are the semiclassical predictions $\bmod (\sigma \pi / 2-\beta, 2 \pi)$, and the data points are calculated using $\bmod (k L+\beta+\gamma, 2 \pi)$. 
(a)

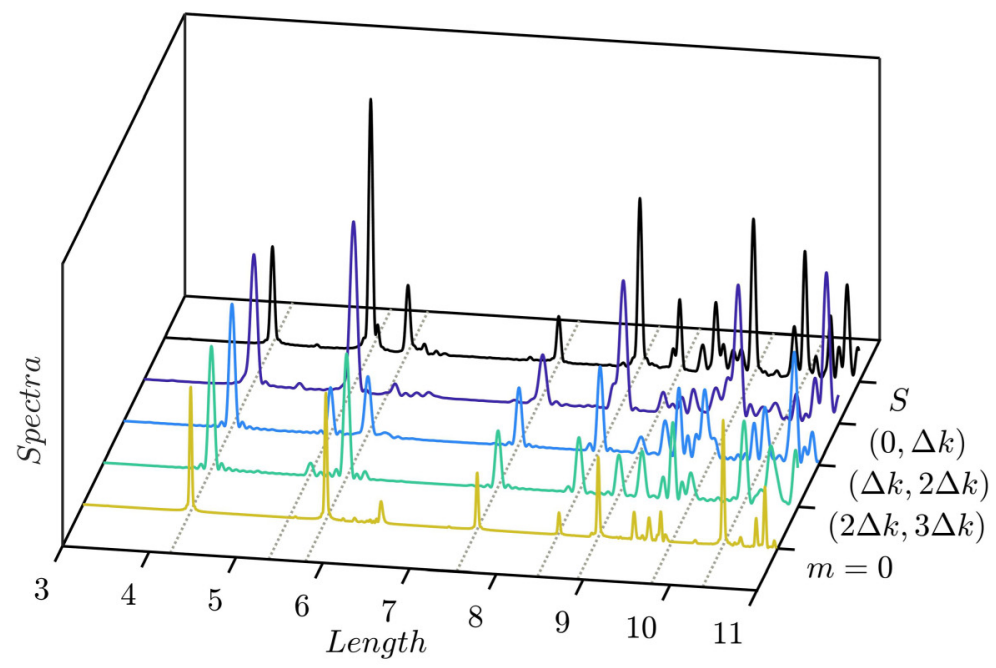

(b)

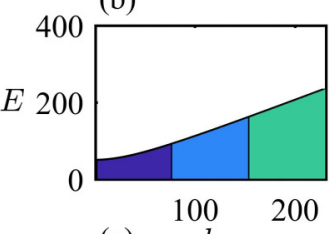

(c)

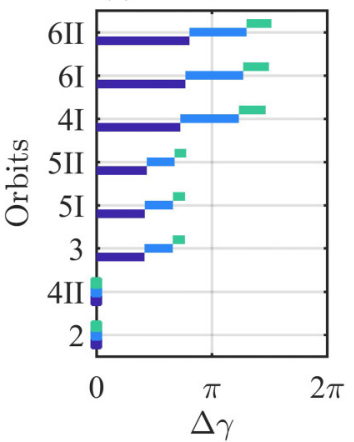

FIG. 14. Length spectra of a heart-sharped chaotic massive Dirac billiard system in different wave-number ranges. (a) Length spectra for $m=50$. The data from back to front correspond to the Schrödinger limit, wave-number range $(0, \Delta k),(\Delta k, 2 \Delta k)$ and, $(2 \Delta k, 3 \Delta k)$, and the massless Dirac limit $(m=0)$, respectively. (b) The three different wave-number ranges. (c) The corresponding difference in the dynamical phase $\Delta \gamma$ for different types of scars. The color scheme is the same as that in panel (b).

can also be calculated from $\bmod (\sigma \pi / 2-\beta, 2 \pi)$ that is independent of $k$ and $m$ but only depends on the geometry of the orbit. By removing the effects of $\gamma, \tilde{\Gamma}$ is a constant independent of $k$ and $m$. Examples of the behaviors of $\tilde{\Gamma}$ for several period-3 scars with different masses are shown in Fig. 13. Since both $\beta$ and $\gamma$ depend on the orientation, $\tilde{\Gamma}^{+}$and $\tilde{\Gamma}^{-}$take on different values, with their difference being $\pi$.

\section{APPENDIX E: ADDITIONAL ANALYSIS OF LENGTH SPECTRA}

Since the dynamical phase in the reflection coefficient is related to $\kappa$ in that increasing the mass is equivalent to decreasing the wave number, it is insightful to investigate the length spectra for eigenwave numbers in different ranges, as shown in Fig. 14. In particular, Fig. 14(c) shows the values
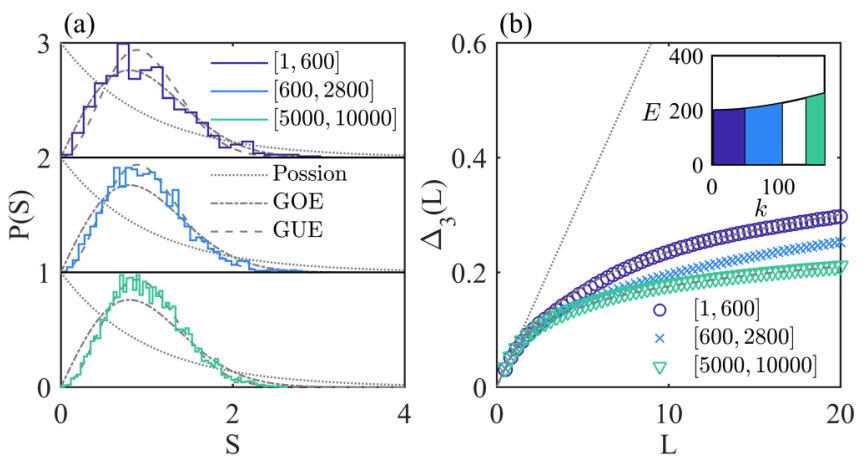

FIG. 15. Energy level spacing distributions for the Africa-shaped massive Dirac billiard in different energy ranges. The same color indicates the same range. The mass value is $m=200$. (a) Illustration of the level spacing statistics in three energy ranges. (b) The corresponding spectral rigidity $\Delta(L)$. Inset shows the corresponding ranges in the $E-k$ plot. of $\Delta \gamma$ in three different wave-number ranges for different orbits with $m=50$. For orbits 2 and 4II, since their current directions are indistinguishable, $\Delta \gamma$ remains to be zero, regardless of the $k$ ranges. It can be seen that, for odd orbits 3, 5I and 5II, as $k$ increases, $\Delta \gamma$ increases from zero to nearly $\pi$ (the massless limit value is $\pi$ ), leading to an increasing difference in the phase between the counterclockwise and clockwise orbits. This effect is stronger in the region of larger wave number, giving rise to a reduction in the peak heights of the length spectrum at the positions of the odd orbits. This can be seen in Fig. 14(a), and the peaks for the odd orbits disappear completely in the massless limit. For the even orbits, e.g., orbits 4I, 6I, and 6II, as $k$ increases, $\Delta \gamma$ increases from zero to about $2 \pi$. For the intermediate wave-number range, $\Delta \gamma$ is close to $\pi$, resulting in a $\pi$ phase difference between the counterclockwise and clockwise current states and leading to the disappearance of the peaks. As a result, the peak height first decreases and then increases with $k$.

\section{APPENDIX F: STATISTICS OF ENERGY LEVEL SPACING FOR DIFFERENT ENERGY RANGES}

An additional result is that, since the dominant factor is not the absolute value of the mass but rather the relative fraction of the energy due to the static mass, for fixed finite mass, varying the wave number $k$ will lead to different phenomena. In particular, for near-zero $k$ values, e.g., for the first few eigenstates, the system is similar to one in the infinite mass limit. For large values of $k$, especially for $\hbar k \gg m c$, the system approaches the massless limit. Thus, when $k$ is varied from small to large values, the level spacing statistics will change from the GOE to the GUE type, as shown in Fig. 15, where the nearest neighbor level spacing probability $P(S)$ and the spectral rigidity $\Delta(L)$ are presented for three different energy ranges for $m=200$. In the low-energy region where the dispersion relation is quadratic, the statistics are 
close to that of GOE. As the energy is increased, the dispersion relation becomes increasingly linear, so the behavior gradually approaches that of massless limit described by the GUE statistics.
[1] H.-J. Stöckmann, Quantum Chaos: An Introduction (Cambridge University Press, New York, 2006).

[2] F. Haake, Quantum Signatures of Chaos, 3rd ed., Springer Series in Synergetics (Springer-Verlag, Berlin, 2010).

[3] S. W. McDonald and A. N. Kaufman, Spectrum and Eigenfunctions for a Hamiltonian with Stochastic Trajectories, Phys. Rev. Lett. 42, 1189 (1979).

[4] S. W. McDonald and A. N. Kaufman, Wave chaos in the stadium: Statistical properties of short-wave solutions of the Helmholtz equation, Phys. Rev. A 37, 3067 (1988).

[5] E. J. Heller, Bound-State Eigenfunctions of Classically Chaotic Hamiltonian Systems: Scars of Periodic Orbits, Phys. Rev. Lett. 53, 1515 (1984).

[6] B. V. Chirikov, An example of chaotic eigenstates in a complex atom, Phys. Lett. A 108, 68 (1985).

[7] R. L. Waterland, J. M. Yuan, C. C. Martens, R. E. Gillilan, and W. P. Reinhardt, Classical-Quantum Correspondence in the Presence of Global Chaos, Phys. Rev. Lett. 61, 2733 (1988).

[8] E. B. Bogomolny, Smoothed wave-functions of chaotic quantum systems, Phys. D (Amsterdam) 31, 169 (1988).

[9] M. V. Berry, Quantum scars of classical closed orbits in phasespace, Proc. R. Soc. London A 423, 219 (1989).

[10] B. Eckhardt, G. Hose, and E. Pollak, Quantum-mechanics of a classically chaotic system: Observations on scars, periodicorbits, and vibrational adiabaticity, Phys. Rev. A 39, 3776 (1989).

[11] R. V. Jensen, M. M. Sanders, M. Saraceno, and B. Sundaram, Inhibition of Quantum Transport Due to Scars of Unstable Periodic Orbits, Phys. Rev. Lett. 63, 2771 (1989).

[12] H. J. Stockmann and J. Stein, Quantum Chaos in Billiards Studied by Microwave Absorption, Phys. Rev. Lett. 64, 2215 (1990).

[13] B. Eckhardt, J. M. G. Llorente, and E. Pollak, Phase-space analysis of chaotic spectra in a conservative Hamiltonian system, Chem. Phys. Lett. 174, 325 (1990).

[14] R. Blümel, I. H. Davidson, W. P. Reinhardt, H. Lin, and M. Sharnoff, Quasi-linear ridge structures in water-surface waves, Phys. Rev. A 45, 2641 (1992).

[15] M. Kus, J. Zakrzewski, and K. Zyczkowski, Quantum scars on a sphere, Phys. Rev. A 43, 4244 (1991).

[16] R. V. Jensen, Quantum chaos, Nature (London) 355, 311 (1992).

[17] R. V. Jensen, Quantum mechanics: Bringing order out of chaos, Nature (London) 355, 591 (1992)

[18] O. Agam and S. Fishman, Quantum eigenfunctions in terms of periodic orbits of chaotic systems, J. Phys. A 26, 2113 (1993).

[19] O. Agam and S. Fishman, Semiclassical Criterion for Scars in Wave Functions of Chaotic Systems, Phys. Rev. Lett. 73, 806 (1994).

[20] G. G. de Polavieja, F. Borondo, and R. M. Benito, Scars in Groups of Eigenstates in a Classically Chaotic System, Phys. Rev. Lett. 73, 1613 (1994).

[21] B. Eckhardt, S. Fishman, J. Keating, O. Agam, J. Main, and K. Müller, Approach to ergodicity in quantum wave functions, Phys. Rev. E 52, 5893 (1995).
[22] T. S. Monteiro, D. Delande, and J. P. Connerade, Have quantum scars been observed? Nature (London) 387, 863 (1997).

[23] C. P. Malta, M. A. M. de Aguiar, and A. M. Ozorio de Almeida, Quantum signature of a period-doubling bifurcation and scars of periodic orbits, Phys. Rev. A 47, 1625 (1993).

[24] T. M. Fromhold, P. B. Wilkinson, F. W. Sheard, L. Eaves, J. Miao, and G. Edwards, Manifestations of Classical Chaos in the Energy-Level Spectrum of a Quantum Well, Phys. Rev. Lett. 75, 1142 (1995).

[25] P. Bellomo and T. Uzer, Quantum scars and classical ghosts, Phys. Rev. A 51, 1669 (1995).

[26] O. Agam, Quantum scars of classical orbits in small interacting electronic systems, Phys. Rev. B 54, 2607 (1996).

[27] S. Fishman, B. Georgeot, and R. E. Prange, Fredholm method for scars, J. Phys. A 29, 919 (1996).

[28] R. Akis, D. K. Ferry, and J. P. Bird, Wave Function Scarring Effects in Open Stadium Shaped Quantum Dots, Phys. Rev. Lett. 79, 123 (1997).

[29] F. P. Simonotti, E. Vergini, and M. Saraceno, Quantitative study of scars in the boundary section of the stadium billiard, Phys. Rev. E 56, 3859 (1997).

[30] L. Kaplan and E. J. Heller, Linear and nonlinear theory of eigenfunction scars, Ann. Phys. 264, 171 (1998).

[31] E. E. Narimanov and A. D. Stone, Origin of Strong Scarring of Wave Functions in Quantum Wells in a Tilted Magnetic Field, Phys. Rev. Lett. 80, 49 (1998).

[32] L. Kaplan, Scars in quantum chaotic wave functions, Nonlinearity 12, R1 (1999).

[33] O. M. Auslaender and S. Fishman, Exact eigenfunctions of a chaotic system, Phys. D (Amsterdam, Neth.) 128, 180 (1999).

[34] J. P. Keating and S. D. Prado, Orbit bifurcations and the scarring of wave functions, Proc. R. Soc. London A 457, 1855 (2001).

[35] B. Huckestein, R. Ketzmerick, and C. H. Lewenkopf, Quantum Transport Through Ballistic Cavities: Soft vs Hard Quantum Chaos, Phys. Rev. Lett. 84, 5504 (2000).

[36] H. Schanz and T. Kottos, Scars on Quantum Networks Ignore the Lyapunov Exponent, Phys. Rev. Lett. 90, 234101 (2003).

[37] D. A. Wisniacki, E. Vergini, R. M. Benito, and F. Borondo, Scarring by Homoclinic and Heteroclinic Orbits, Phys. Rev. Lett. 97, 094101 (2006)

[38] A. Bäcker, R. Ketzmerick, S. Löck, M. Robnik, G. Vidmar, R. Höhmann, U. Kuhl, and H.-J. Stöckmann, Dynamical Tunneling in Mushroom Billiards, Phys. Rev. Lett. 100, 174103 (2008).

[39] P. J. J. Luukko, B. Drury, A. Klales, L. Kaplan, E. J. Heller, and E. Räsänen, Strong quantum scarring by local impurities, Sci. Rep. 6, 37656 (2016).

[40] K. S. Novoselov, A. K. Geim, S. V. Morozov, D. Jiang, Y Zhang, S. V. Dubonos, I. V. Grigorieva, and A. A. Firsov, Electric field effect in atomically thin carbon films, Science 306 , 666 (2004).

[41] C. Berger, Z. M. Song, T. B. Li, X. B. Li, A. Y. Ogbazghi, R. F. Z. T. Dai, A. N. Marchenkov, E. H. Conrad, P. N. First, and W. A. de Heer, Ultrathin epitaxial graphite: 2D electron gas 
properties and a route toward graphene-based nanoelectronics, J. Phys. Chem. B 108, 19912 (2004).

[42] K. S. Novoselov, A. K. Geim, S. V. Morozov, D. Jiang, M. I. Katsnelson, I. V. Grigorieva, S. V. Dubonos, and A. A. Firsov, Two-dimensional gas of massless Dirac fermions in graphene, Nature (London) 438, 197 (2005).

[43] Y. B. Zhang, Y. W. Tan, H. L. Stormer, and P. Kim, Experimental observation of the quantum Hall effect and Berry's phase in graphene, Nature (London) 438, 201 (2005).

[44] A. H. Castro Neto, F. Guinea, N. M. R. Peres, K. S. Novoselov, and A. K. Geim, The electronic properties of graphene, Rev. Mod. Phys. 81, 109 (2009).

[45] N. M. R. Peres, Colloquium: The transport properties of graphene: An introduction, Rev. Mod. Phys. 82, 2673 (2010).

[46] S. Das Sarma, S. Adam, E. H. Hwang, and E. Rossi, Electronic transport in two-dimensional graphene, Rev. Mod. Phy. 83, 407 (2011).

[47] Y.-C. Lai, H.-Y. Xu, L. Huang, and C. Grebogi, Relativistic quantum chaos: An emergent interdisciplinary field, Chaos $\mathbf{2 8}$, 052101 (2018).

[48] L. Huang, H.-Y. Xu, C. Grebogi, and Y.-C. Lai, Relativistic quantum chaos, Phys. Rep. 753, 1 (2018).

[49] M. V. Berry and R. J. Mondragon, Neutrino billiards: Timereversal symmetry-breaking without magnetic-fields, Proc. R. Soc. London A 412, 53 (1987).

[50] L. Huang, Y.-C. Lai, D. K. Ferry, S. M. Goodnick, and R. Akis, Relativistic Quantum Scars, Phys. Rev. Lett. 103, 054101 (2009).

[51] D. Cabosart, A. Felten, N. Reckinger, A. Iordanescu, S. Toussaint, S. Faniel, and B. Hackens, Recurrent quantum scars in a mesoscopic graphene ring, Nano Lett. 17, 1344 (2017).
[52] H. Y. Xu, L. Huang, Y.-C. Lai, and C. Grebogi, Chiral Scars in Chaotic Dirac Fermion Systems, Phys. Rev. Lett. 110, 064102 (2013).

[53] X. Ni, L. Huang, Y.-C. Lai, and C. Grebogi, Scarring of Dirac fermions in chaotic billiards, Phys. Rev. E 86, 016702 (2012).

[54] L. Huang, H.-Y. Xu, Y.-C. Lai, and C. Grebogi, Level spacing statistics for two-dimensional massless Dirac billiards, Chin. Phys. B 23, 070507 (2014).

[55] C.-Z. Wang, L. Huang, and K. Chang, Scars in Dirac fermion systems: The influence of an Aharonov-Bohm flux, New J. Phys. 19, 013018 (2017).

[56] M. Z. Hasan and C. L. Kane, Colloquium: Topological insulators, Rev. Mod. Phys. 82, 3045 (2010)

[57] X.-L. Qi and S.-C. Zhang, Topological insulators and superconductors, Rev. Mod. Phys. 83, 1057 (2011).

[58] Y. Zhang, T. Tang, C. Girit, Z. Hao, M. C. Martin, A. Zettl, M. F. Crommie, Y. R. Shen, and F. Wang, Direct observation of a widely tunable bandgap in bilayer graphene, Nature (London) 459, 820 (2009).

[59] M. Robnik, Quantizing a generic family of billiards with analytic boundaries, J. Phys. A 17, 1049 (1984).

[60] H.-Y. Xu, L. Huang, Y.-C. Lai, and C. Grebogi, Superpersistent currents and whispering gallery modes in relativistic quantum chaotic systems, Sci. Rep. 5, 8963 (2015).

[61] J. Samuel and R. Bhandari, General Setting for Berry's Phase, Phys. Rev. Lett. 60, 2339 (1988).

[62] S.-J. Choi, S. Park, and H.-S. Sim, Tunable geometric phase of Dirac fermions in a topological junction, Phys. Rev. B 87, 165420 (2013). 